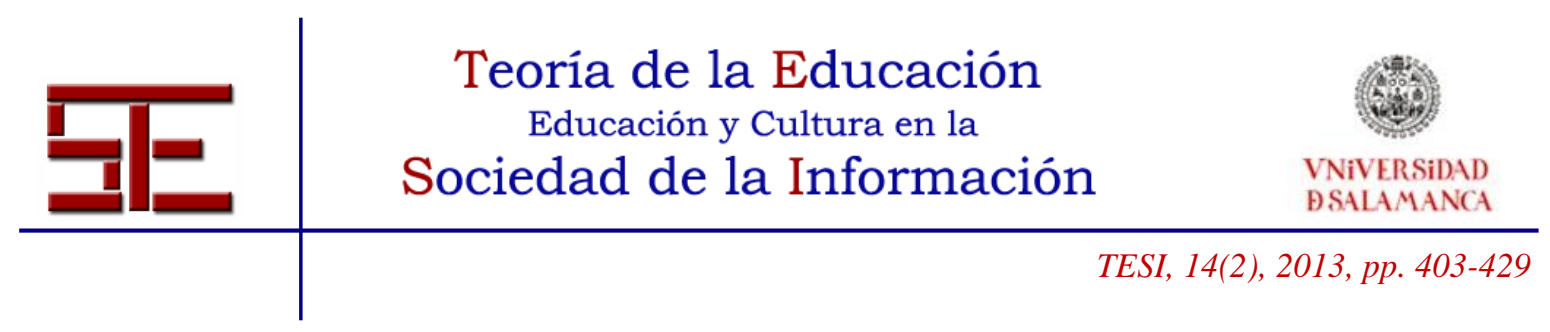

\title{
ANÁLISIS DE LA EXPERIENCIA DE USO DE UN AGENTE DE COMPRENSIÓN LECTORA CON NIÑOS EN EDAD ESCOLAR
}

Resumen: El papel de las tecnologías en la sociedad es fundamental y en continuo crecimiento. En el contexto educativo, se pueden identificar en las últimas décadas un incremento de nuevas tecnologías educativas, entre las que se encuentran el uso de Agentes Conversacionales Pedagógicos. Esto es, sistemas informáticos que interaccionan con los estudiantes en lenguaje natural, hablado o escrito, y que pueden adoptar el rol de profesor, estudiante o bien compañero de estudios.

En el curso 2011/2012, aplicamos el Agente Conversacional Pedagógico llamado Dr. Roland, para resolver problemas de matemáticas, en un aula con 38 estudiantes de entre 12-13 años de edad y 3 profesores. En este artículo, se presenta el análisis de la experiencia y cómo se pueden integrar estas nuevas tecnologías en el aula.

También se proporcionan, a partir del análisis realizado, diez recomendaciones que se proponen como guía para docentes, investigadores y profesionales interesados en usar este tipo de agentes. Algunas de estas recomendaciones son combinar el uso del ordenador, con otros dispositivos como tabletas que pueden facilitar el manejo del sistema por varios estudiantes, incluir a los padres para fomentar el uso del agente desde casa, y validar cada uno de los avances realizados con los profesores, padres y estudiantes para adaptarse a sus necesidades.

Palabras clave: Agente Conversacional Pedagógico; Enseñanza Escolar; Diseño Centrado en el Usuario; Interacción en Lenguaje Natural.

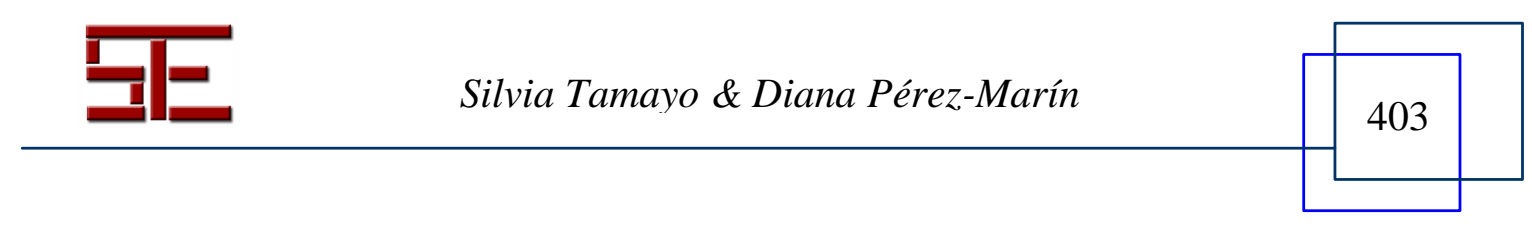




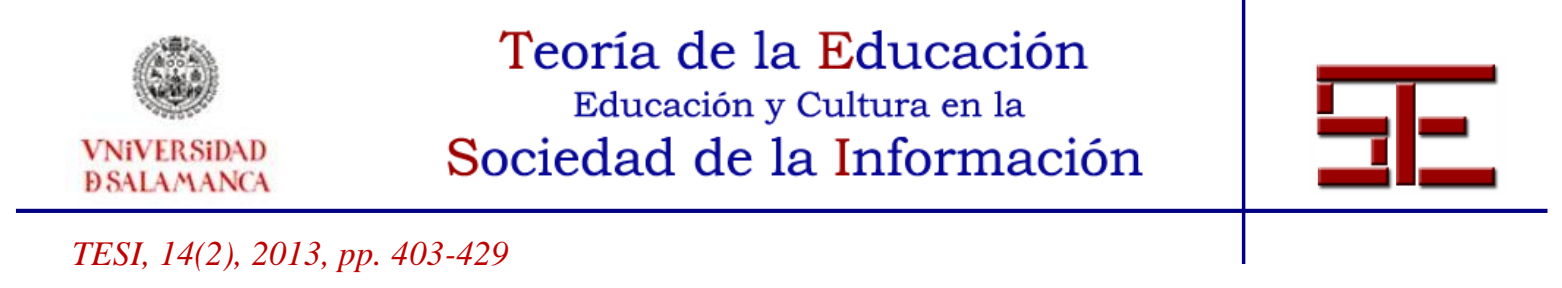

\title{
ANALYSIS OF THE EXPERIENCE OF USING A PEDAGOGIC CONVERSATIONAL AGENT FOR READING COMPREHENSION IN THE SCHOOL
}

\begin{abstract}
Technologies are key in society, and they are in continuous progress. In the educational context, an increase of using new technologies for education can be identified in the last years. One of these new technologies is Pedagogic Conversational Agents. That is, computer systems that interact with the students in spoken or written natural language. They can adopt the role of teacher, student or pal.

In the 2011/2012 academic year, we used a Pedagogic Conversational Agent called Dr. Roland in class to solve Maths problems. 38 students and 3 teachers were involved in the experience. In this paper, an analysis of the experience and how to integrate agents in the school is presented.

Ten recommendations as a guide for teachers, researchers and developers interested in using this type of educational software are proposed from the analysis. Some of these recommendations are to combine the use of computers with other devices such as tablets that make the use of the system by several students easier, to involve the parents to foster the use of the agent at home, and to validate each step of the advances made with teachers, parents and students so that they are adapted to their needs.
\end{abstract}

Key words: Pedagogic Conversational Agent; School Education; User-Centered Design; Natural Language Interaction.

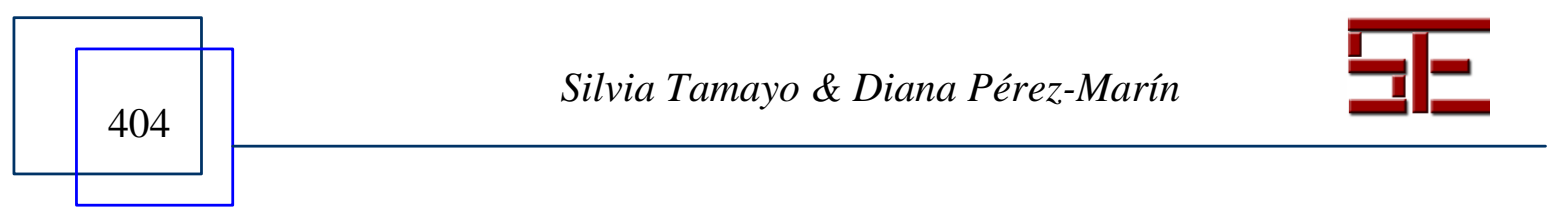




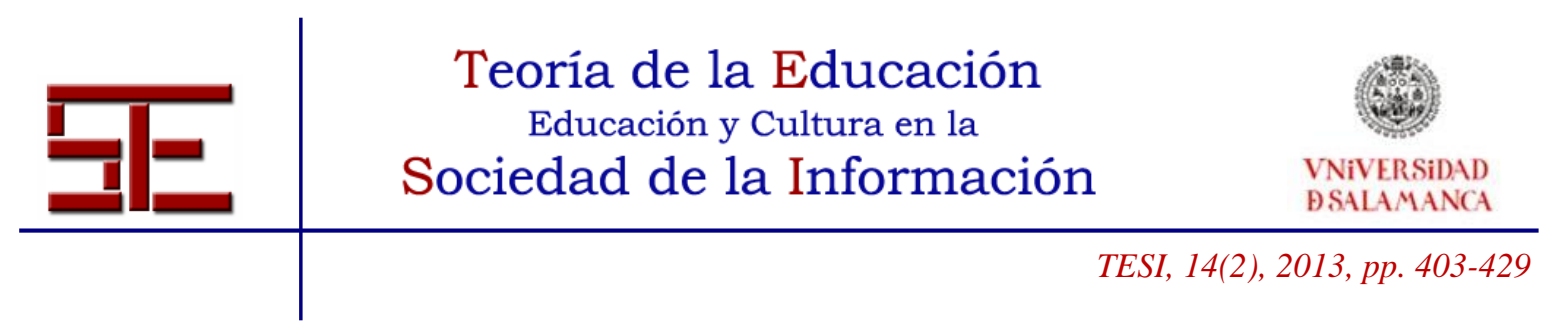

\section{ANÁLISIS DE LA EXPERIENCIA DE USO DE UN AGENTE DE COMPRENSIÓN LECTORA CON NIÑOS EN EDAD ESCOLAR}

Fecha de recepción: 22/01/2013; fecha de aceptación: 15/04/2013; fecha de publicación: 30/07/2013

Silvia Tamayo

s.tamayo@alumnos.urjc.es

Universidad Rey Juan Carlos

Diana Pérez-Marín

diana.perez@urjc.es

Universidad Rey Juan Carlos

\section{INTRODUCCIÓN}

Las nuevas tecnologías son un eje fundamental en la sociedad con una influencia cada vez mayor en todos los contextos. En particular, en el contexto educativo se están utilizando tecnologías como sistemas de Realidad Aumentada, Pizarras Digitales, Webquests en Internet, Agentes Conversacionales Pedagógicos, etc. que proporcionan múltiples beneficios (Dussel et al. 2011). En particular, los Agentes Pedagógicos Conversacionales, son sistemas interactivos que permiten a los estudiantes repasar de una forma entretenida y amigable evitando los problemas de falta de motivación que a veces registran otros métodos educativos (Johnson et al. 2000).

Los agentes proporcionan además la ventaja de poder interactuar en lenguaje natural con los estudiantes, facilitando de esta forma su manejo. Esto es importante para que los estudiantes puedan concentrarse en la tarea a realizar en lugar de tener que aprender cómo manejar el sistema según los principios de la Interacción Persona-Ordenador (Nielsen, 1993).

En la actualidad, existen cientos de agentes distintos (Pérez-Marín \& Pascual-Nieto, 2011) aplicados en dominios muy diversos desde la enseñanza de Ciencias Naturales (Lester et al. 1997) hasta Idiomas (Reategui et al. 2007) o competencias como la

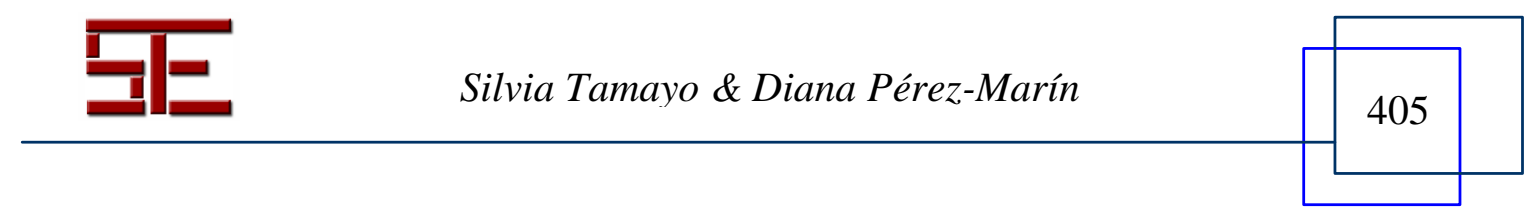




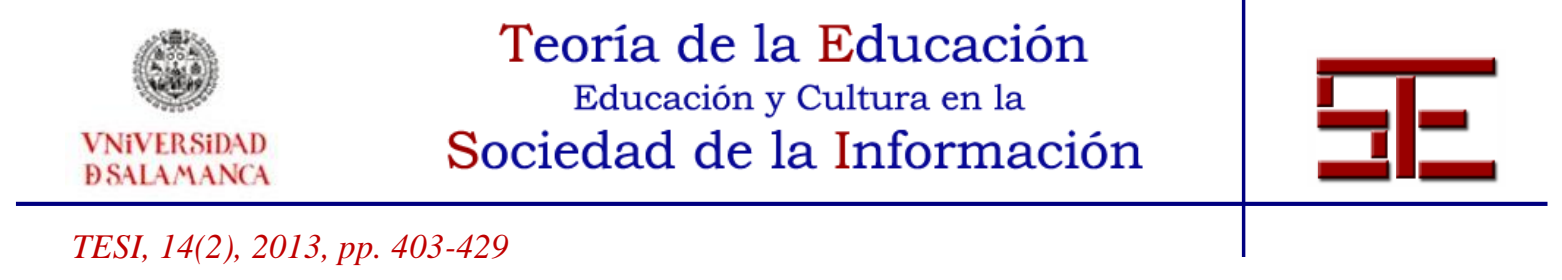

capacidad de contar cuentos (Ryokai et al. 2003) o empatizar con otras culturas (Hays et al. 2009).

Algunos de los beneficios que se han estudiado del uso de este tipo de agentes son: el efecto Persona (Lester et al. 1997) según el cual la mera presencia del agente en el entorno educativo puede tener un efecto positivo en la percepción de la experiencia educativa por parte del estudiante; el efecto Proteo (Yee \& Bailenson, 2007) según el cual los estudiantes pueden aprender motivados por conseguir las características de sus agentes y parecerse a ellos; y, el efecto Protégé (Chase et al. 2009) según el cual los estudiantes pueden hacer un esfuerzo mayor para enseñar a su agente que para aprender ellos mismos.

En este artículo, se quiere analizar qué efectos tiene el uso de un agente para enseñar matemáticas cuando se integra su uso en el aula, y concluir un conjunto de recomendaciones que sirvan de guía para cualquier profesor, investigador o profesional que quiera utilizar esta prometedora tecnología educativa.

Se ha escogido el dominio de las matemáticas porque para poder resolver problemas de matemáticas, es necesario tener competencias transversales como la capacidad de lectura de los ejercicios (Baroody, 1987) y superar posibles bloqueos emocionales por temor a no poder resolverlos (Wright, 1996; Ginsburg, 1977).

La Figura 1 muestra un pantallazo del agente al que se ha llamado Dr. Roland. Se ha desarrollado según un Diseño Centrado en el Usuario teniendo en cuenta las necesidades y requisitos solicitados por los profesores y los estudiantes desde el principio y durante todo su desarrollo (Tamayo \& Pérez-Marín, 2012a).

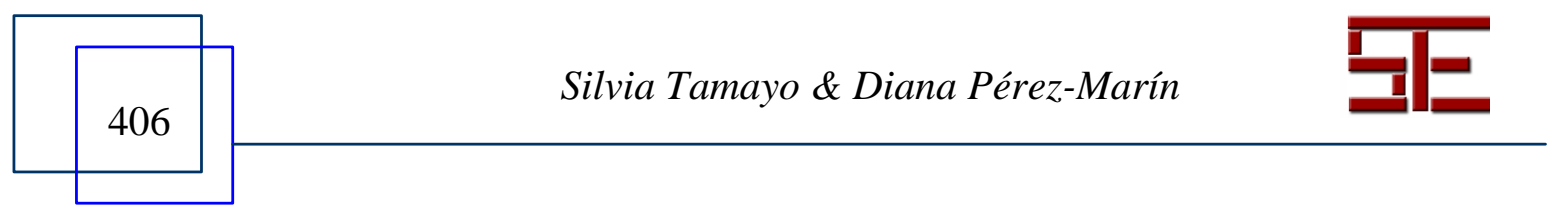



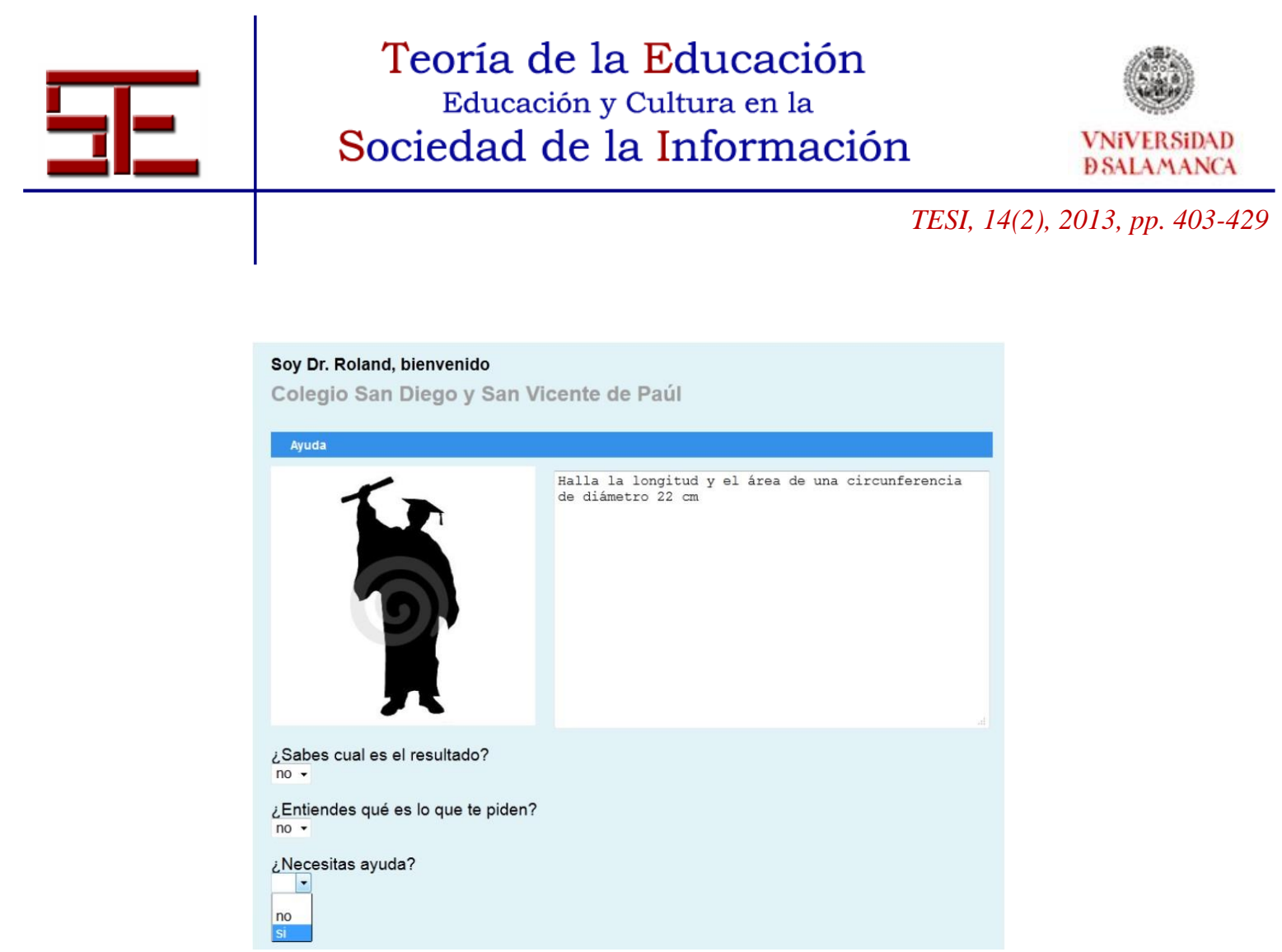

Figura 1. Ejemplo de pantalla de Dr. Roland

Dr. Roland ha sido utilizado durante el curso 2011/2012 en un colegio en Madrid, en el que han participado 38 estudiantes y 3 profesores. El algoritmo RUANLP es el responsable de guiar el diálogo entre el estudiante y el sistema para poder resolver los ejercicios de matemáticas (Tamayo \& Pérez-Marín, 2012b). Se partió de tres hipótesis:

H1. Es posible desarrollar un Agente de Comprensión Lectora para soporte de problemas de matemáticas utilizando Diseño Centrado en el Usuario.

H2. Es posible que haya una relación entre el uso del agente y la capacidad de resolución o comprensión de los ejercicios por parte de los estudiantes en edad escolar.

H3. Es posible obtener un alto nivel de satisfacción/ aceptación por parte de los usuarios al emplear el agente.

Hay que tener en cuenta, no obstante, que debido a las limitaciones de planificación en los colegios, los estudiantes no pudieron utilizar en clase Dr. Roland durante todo el curso, sino solo los días indicados por los profesores. Tampoco utilizaron el sistema via web, aunque se permitió, posiblemente porque durante la experiencia no se involucró a los padres. En todo caso, los resultados encontrados proporcionan evidencia para las

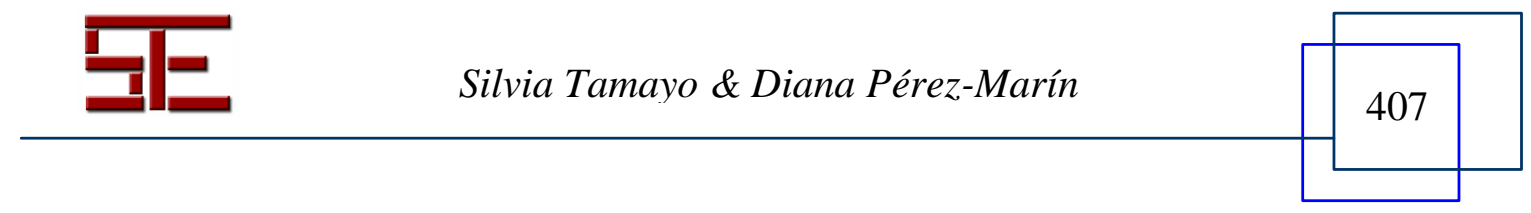




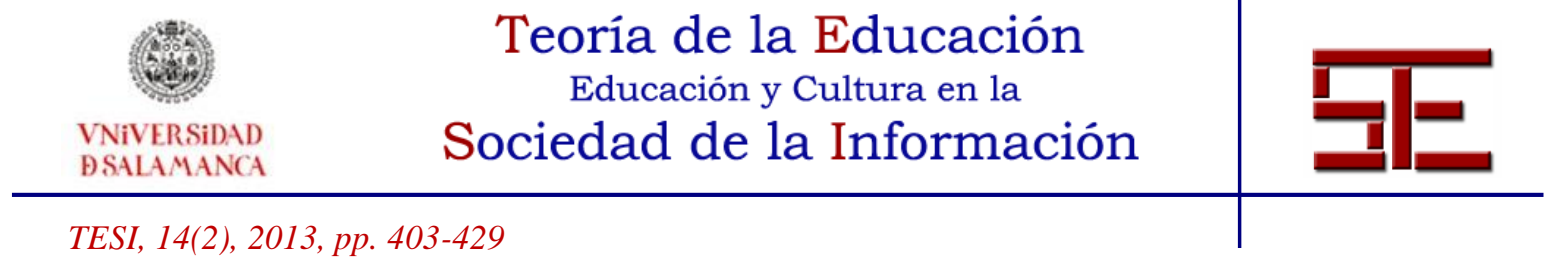

hipótesis $\mathrm{H} 1$ y H3. Se ha podido desarrollar el agente, usarlo y se ha registrado una mejoría en la capacidad de comprensión de los estudiantes (+0.5 al usar el agente).

Sin embargo, no se encuentra una mejora de la capacidad de resolución de los ejercicios. Esto sugiere que no se puede corroborar la hipótesis $\mathrm{H} 2$ de que si los alumnos comprenden mejor el ejercicio pueden resolverlo mejor. Por su parte, se valida $\mathrm{H} 3$, a partir de los cuestionarios recogidos de los estudiantes donde el 94,7\% de los estudiantes dicen que Dr. Roland les parece muy útil, y el $90 \%$ están absortos en la tarea de resolver los problemas de matemáticas con el agente durante la hora de clase.

A partir de esta experiencia y su análisis, se pueden concluir diez recomendaciones que se proponen como guía para docentes, investigadores y profesionales interesados en usar este tipo de agentes. Algunas de estas recomendaciones son combinar el uso del ordenador, con otros dispositivos como tabletas que pueden facilitar el manejo del sistema por varios estudiantes, incluir a los padres para fomentar el uso del agente desde casa, y validar cada uno de los avances realizados con los profesores, padres y estudiantes para adaptarse a sus necesidades.

La estructura del artículo es la siguiente: el apartado 2 presenta el contexto de investigación donde se enmarca este estudio; el apartado 3 proporciona la descripción del agente; el apartado 4 se centra en la experiencia y su análisis; el apartado 5 termina con las principales conclusiones y recomendaciones que se pueden extraer a partir del análisis de la experiencia realizada con el agente.

\section{2.- ESTADO DEL ARTE}

En los últimos años, se ha desarrollado el uso de agentes conversacionales, que se definen como guías, maestros, ayudantes, la personalidad de la máquina o de un programa específico. En interfaces conversacionales, el uso que se les da es la búsqueda de una interacción más personalizada entre la máquina y el usuario (Mas, 2005).

Entre las características de los agentes está la autonomía, ya que deben ser capaces de responder acorde a los conocimientos que les permitan conseguir el objetivo planteado; proactividad, tomando la iniciativa mediante sugerencias o acciones que les permitan alcanzar su objetivo; sociabilidad puesto que deben ser comunicativos y cooperativos; y tienen que tener una personalidad con un comportamiento adaptado al objetivo que persiguen (Cassell, 2000).

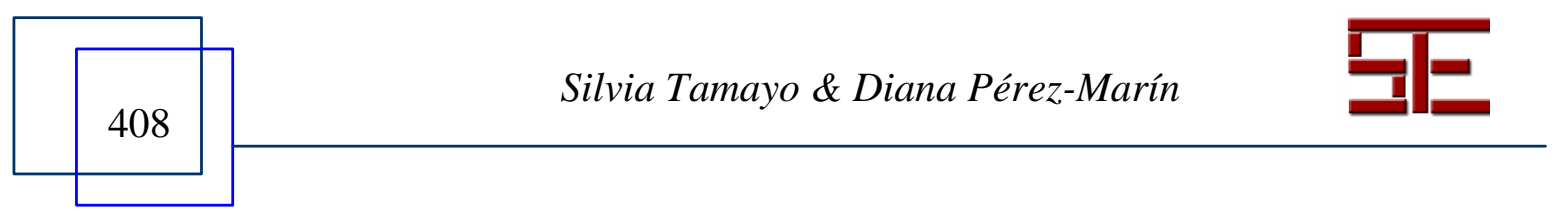




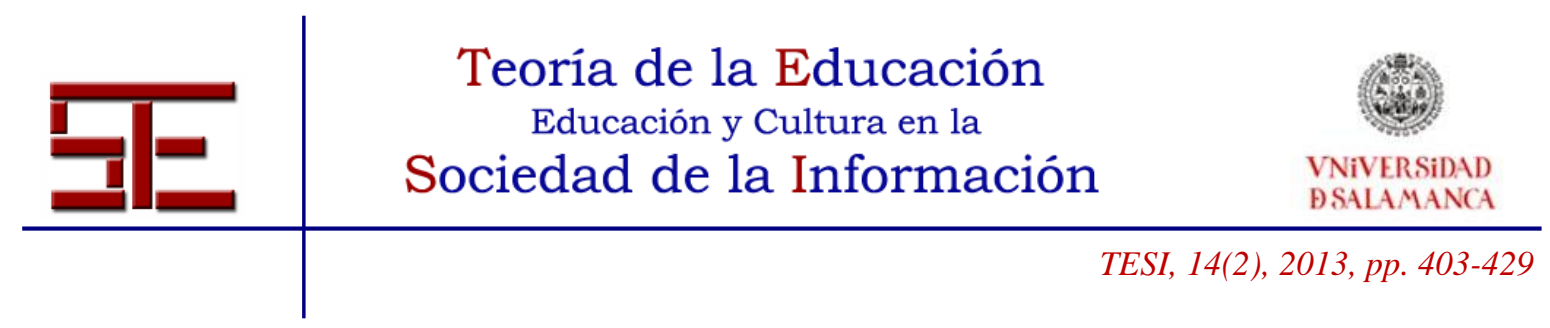

Más concretamente, los agentes animados o conversacionales, son entidades que se pueden representar con personas, animales, cosas que pueden hablar con sonido o texto. Los agentes son un apoyo para una amplia gama de aplicaciones en empresas comerciales, salud, entrenamiento, educación, etc. En el dominio de la educación, que nos ocupa en este artículo, los agentes conversacionales pedagógicos permiten actuar como profesor, estudiante o acompañante del estudiante (Johnson et al. 2000).

\subsection{Técnicas}

Existen diversas técnicas para el funcionamiento de agentes conversacionales, y las categorías principales en las que se clasifican son: aquellas que se basan en el emparejamiento de palabras y frases, y las técnicas estadísticas (Kerly et al. 2008). Las primeras, obtienen las frases de la entrada del usuario, y las emparejen con palabras y frases específicas para categorizar las respuestas del usuario. Mientras que las segundas, tienen que ver con el análisis de la frecuencia y asociaciones de palabras según aparezcan en los textos.

Existen también trabajos que tienen relación con modelos computacionales de diálogo. Sistemas que interaccionan con los usuarios como si se tratase de uno más de la conversación. Generalmente, dichos sistemas están basados en el análisis sistemático del cuerpo del diálogo. Los modelos se pueden realizar atendiendo al nivel de estructura superficial del discurso o profunda del discurso. En relación al primero, son varios los ejemplos existentes, como DAMSL (2012), centrados en la delimitación de los diferentes actos del habla y su trabajo en el desarrollo del diálogo.

En los estudios sobre gestión del diálogo apenas se ha tratado el nivel de estructura profunda. No obstante, algunos ejemplos son el HCRC Dialogue Structure Coding (Carletta et al. 1996), formado por 128 diálogos y 15 horas de conversación que se orienta a la descripción de rutas, o el HCRC Map Task Corpus.

Para el desarrollo de un agente conversacional, las recomendaciones principales a considerar, recopiladas en la literatura del campo son (Kerly et al. 2008):

- En el diseño del sistema tiene implicaciones importantes el alcance del agente conversacional, puesto que depende del rol que va a desempeñar el agente en el sistema, así como de nivel de experiencia. Hay que definir quién controla la dirección de la conversación y quién lleva la iniciativa en el flujo. También, hay que delimitar el nivel de libertad que tendrán los usuarios.

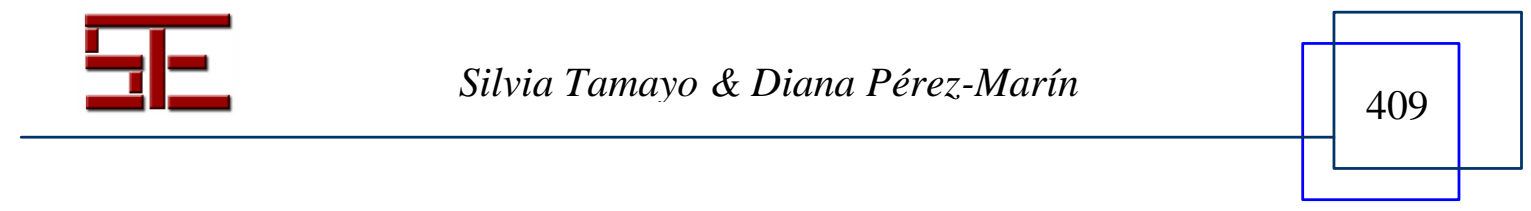




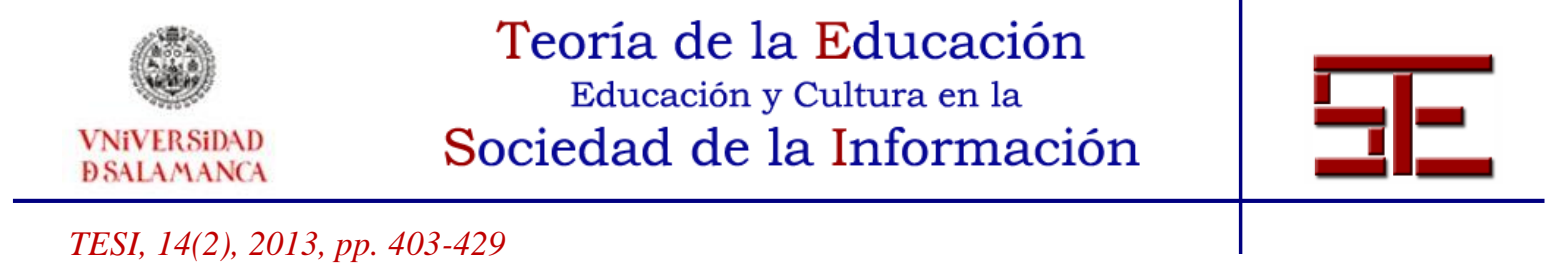

- Se tienen que marcar pautas del procedimiento de los usuarios a través del sistema, marcarles un objetivo, o permitirles navegar por todas las posibilidades que ofrezca el sistema.

- Definir pautas a seguir cuando el usuario inserta preguntas o frases inesperadas. También puede ocurrir que se inserten conceptos que estén expresados de una forma diferente pero con el mismo significado, lo que debe ser considerado por los desarrolladores.

- Realización de análisis de la estructura de los diálogos humano-humano, para obtener información y desarrollar sistemas que puedan interaccionar con las personan en un diálogo.

- Realizar análisis de documentos relacionados con la finalidad de obtener información sobre el contexto.

\subsection{Ejemplos}

Los agentes pueden asumir muchos roles, y se encuentran ejemplos en la literatura de agentes actuando como profesor, estudiante o acompañante. Sin embargo, puesto que en este artículo nos centramos en agentes con el rol de profesor, en este apartado nos limitaremos a mostrar algunos ejemplos de agentes profesor. El primer agente revisado ha sido Autotutor, que se basa en teorías constructivistas, teniendo iniciativa mixta de diálogo y animación 3D (Graesser et al. 2001). Autotutor es un referente en agentes pedagógicos desde los años 90. La Figura 2 muestra un pantallazo de este agente.

En su funcionamiento utiliza Análisis de la Semántica Oculta combinada con técnicas de procesamiento de lenguaje natural (Graesser et al. 2007). Según los creadores de Autotutor la mejora en los resultados finales de los exámenes de los estudiantes que lo utilizan para repasar es de un 0.8 .

En la actualidad, se trabaja en la mejora de la capacidad del agente para la detección de emociones y responder empáticamente al estudiante. Además, se han hecho estudios contrastando la efectividad de mostrar el diálogo únicamente, sin y con la presencia del agente, comprobándose que cuando el agente está presente los resultados son mejores, lo que se conoce como Efecto Persona (Lester et al. 1997).

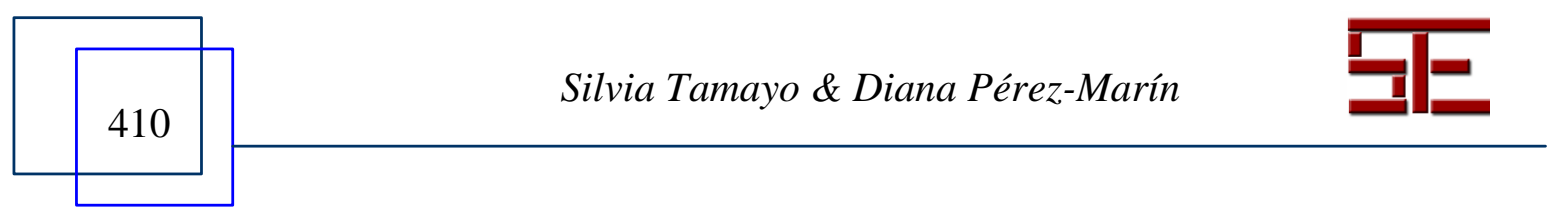



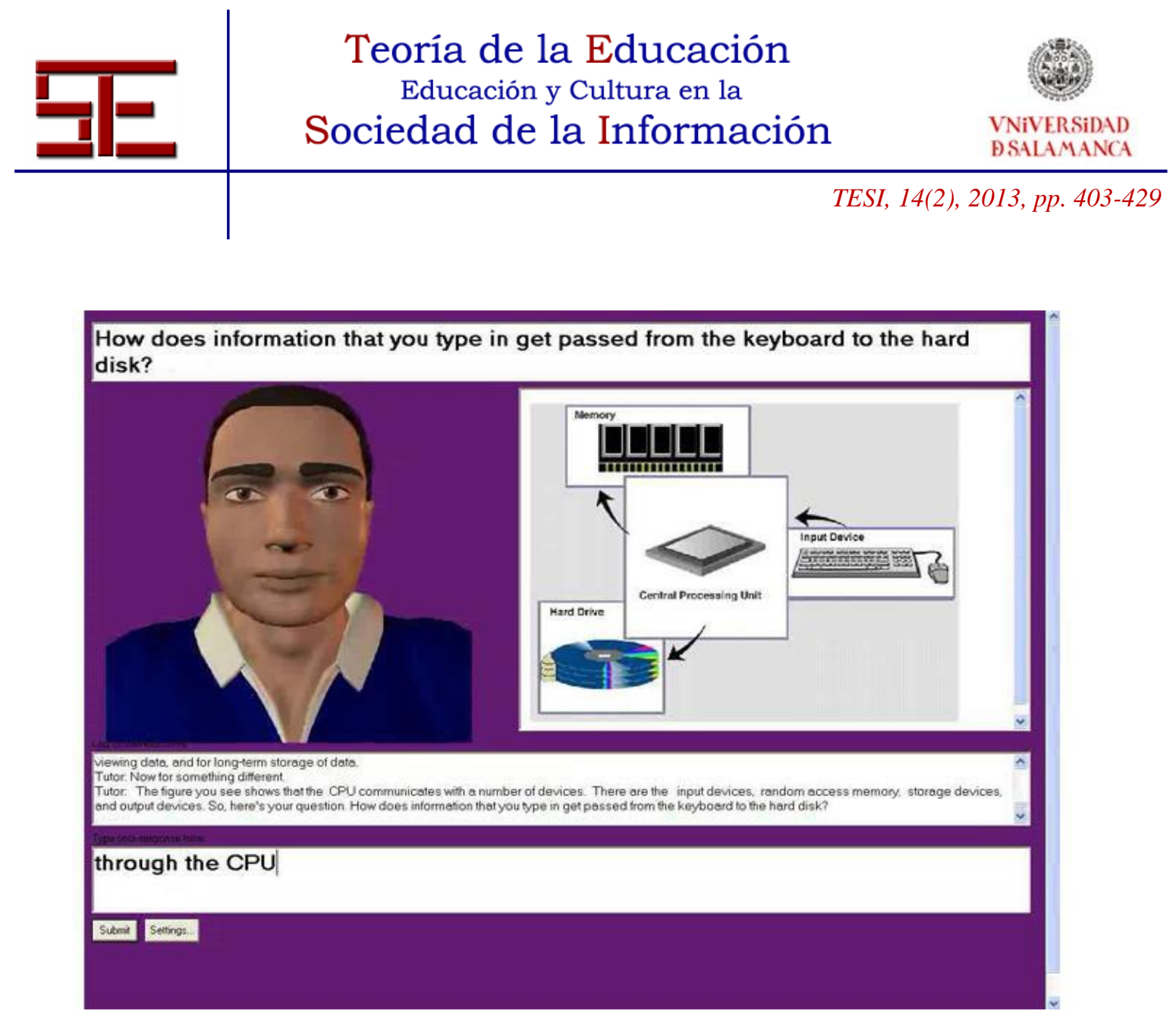

Figura 2. Interfaz del agente Autotutor

Otro ejemplo de agente es Laura que es un agente profesor que se ha empleado para alumnos de un curso de aprendizaje de lengua española. Laura ha sido diseñada para hacer conversaciones con estudiantes mediante texto y diálogos (Theodoridou \& Yerasimou, 2008). Además, cuenta con imágenes animadas de lo que representa y voz para pronunciar palabras. Puede verse una imagen de Laura en la Figura 3.

En cuanto a la experimentación, Laura ha sido probada en EE.UU. en un estudio de investigación universitario. El experimento consistió en un grupo de 23 alumnos que utilizaban Laura solo con voz, y otro grupo de 24 alumnos que utilizaban Laura con la imagen animada y la voz. Laura también incluye un chat en el que los estudiantes pueden practicar vocabulario y enlazarlo en conversaciones con el agente. Un $87.5 \%$ de los estudiantes del primer grupo y un $87 \%$ de los estudiantes del segundo grupo afirmó que Laura les había resultado de ayuda en la mejora de su capacidad de conversar en español.

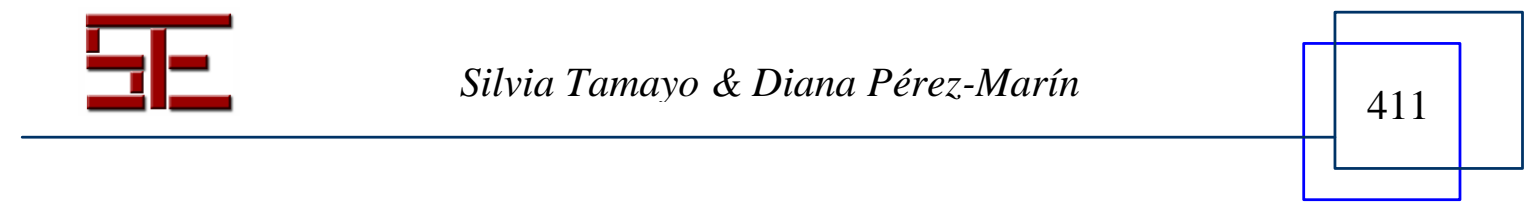




Teoria de la Educación
Educación y Cultura en la
$\begin{gathered}\text { VNIVERSADAD } \\ \text { BSALAMANCA }\end{gathered}$

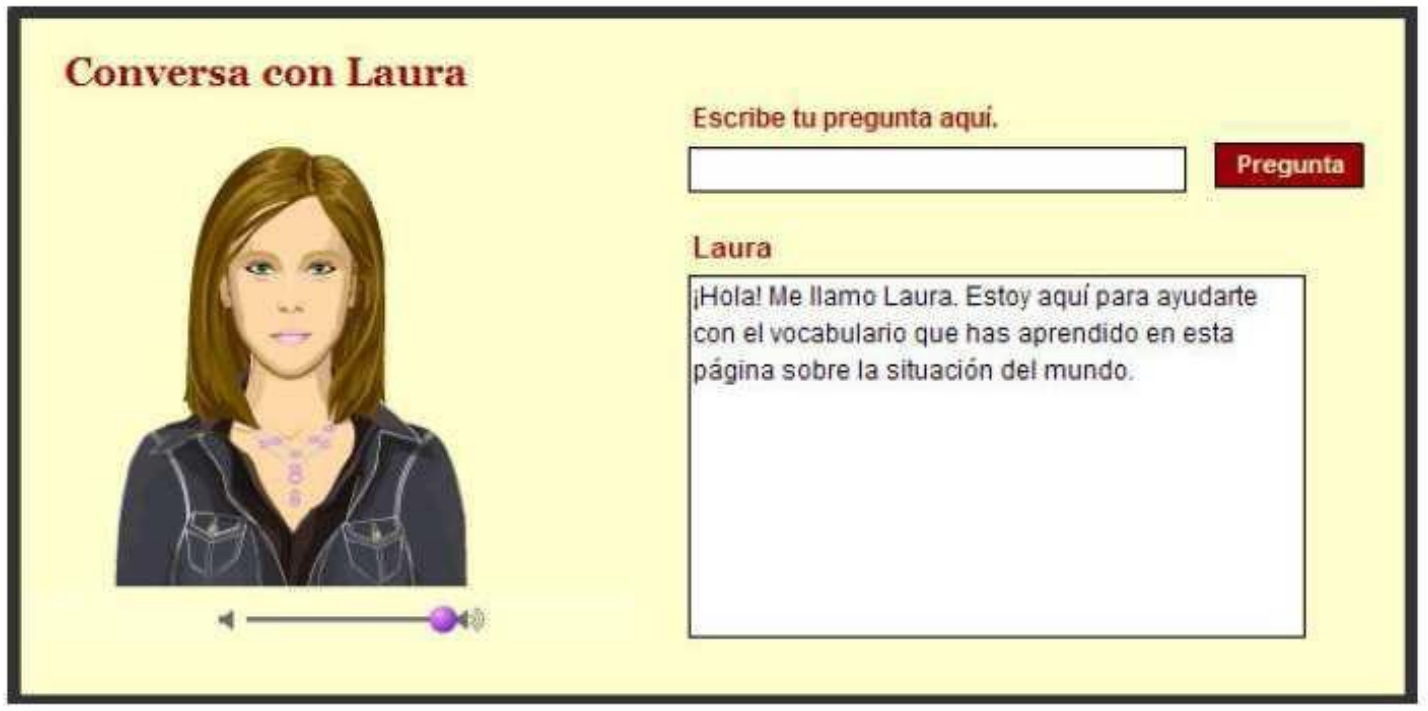

Figura 3. Interfaz del agente Laura

Los estudiantes destacan de Laura su naturaleza interactiva (76.6\%) como aspecto más positivo, y como aspecto negativo la distracción ocasional con elementos multimedia $(12 \%)$. Actualmente, se está plateando la posibilidad de planificar la interacción de Laura de tal forma que atraiga la atención de los estudiantes, y mejore la capacidad de conversación de los estudiantes.

Un último ejemplo de agente profesor, aunque hay muchos otros, pero para mostrar la variedad de técnicas y dominios en los que se están utilizando podría ser Willow (Pérez-Marín, 2007). Se trata de un sistema de evaluación automática y adaptativa de respuestas en texto libre (Pérez-Marín, 2007), pudiendo escribirse en inglés o español, y siendo contrastadas con referencias insertadas por diferentes profesores. Puede verse una imagen de Willow en la Figura 4.

Cabe destacar que tanto el avatar que representa al sistema, como el avatar que representa al estudiante pueden personalizarse. Además, cuando Willow procesa respuestas, muestra retroalimentación inmediata, informa al estudiante del resultado de la respuesta, y muestra las respuestas correctas del profesor.

Willow se ha probado desde el año 2005 con alumnos de diferentes ámbitos, tanto de Ingeniería Informática, como de Telecomunicaciones y de Filología Inglesa,

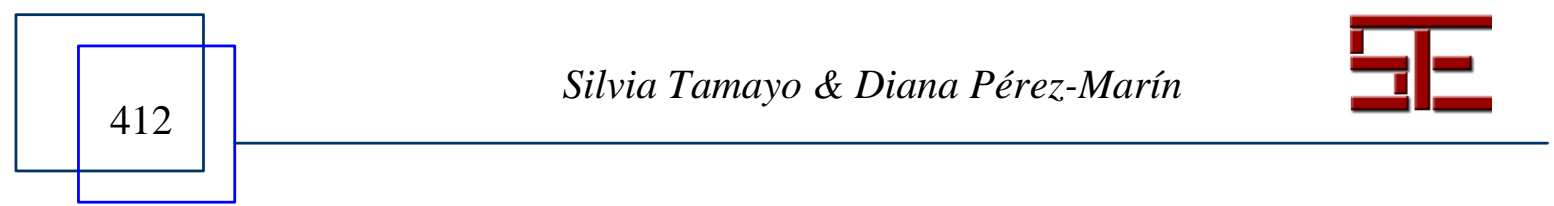




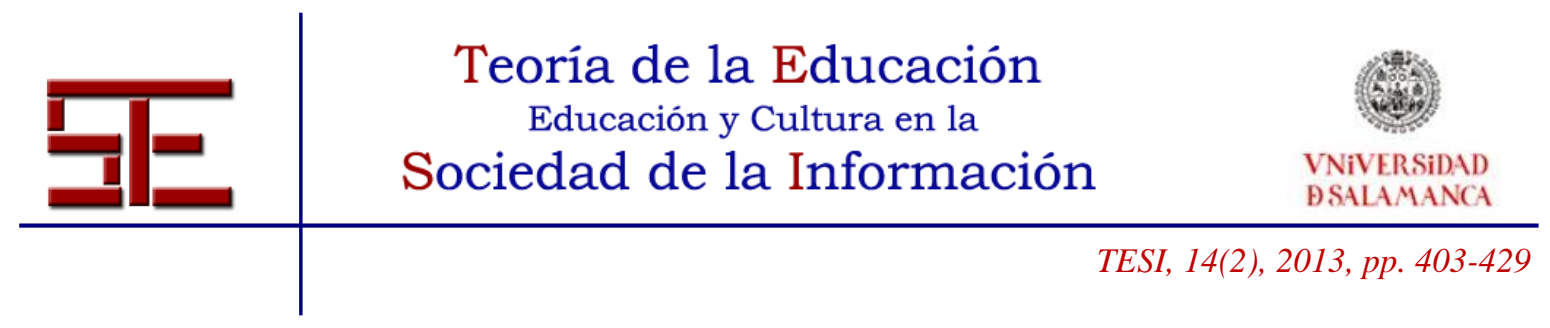

obteniéndose respuestas positivas ante la consulta de la utilidad para el repaso, y siendo usado por los estudiantes de forma regular en el tiempo.

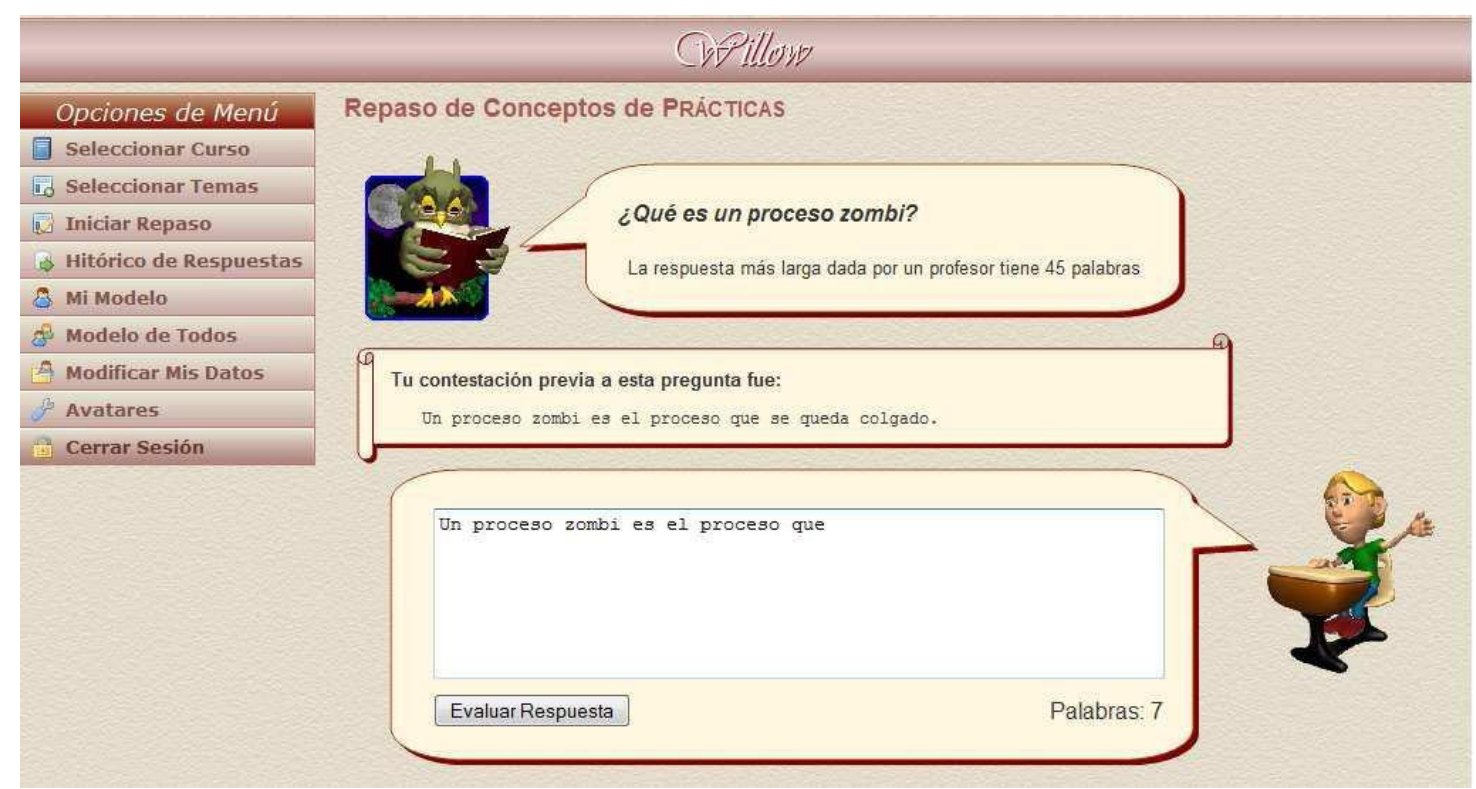

Figura 4. Interfaz de Willow

\section{EL AGENTE DR. ROLAND}

El agente Dr. Roland se desarrolló con el objetivo de ayudar a los estudiantes a resolver problemas de matemáticas, con la hipótesis de que si los estudiantes mejoran la comprensión de ejercicios en el área de matemáticas, también podrán mejorar su capacidad de resolverlos. Desde el principio el agente se desarrolló teniendo en cuenta las necesidades y requisitos que nos pedían los profesores y los estudiantes.

En primer lugar, se comenzó trabajando sobre la aplicación para el profesor. El objetivo era permitir a los profesores que introdujeran problemas, su solución y pistas para facilitar a los estudiantes la comprensión de su enunciado. Los profesores nos pidieron que el sistema permitiera identificar las frases clave de cada enunciado, palabras clave, pronombres y verbos. La Figura 5 muestra un ejemplo de la interfaz de Dr. Roland para permitir a los profesores editar enunciados de los problemas.

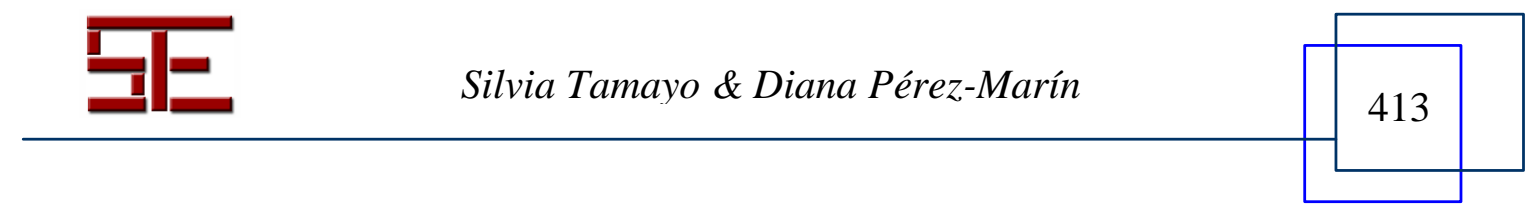




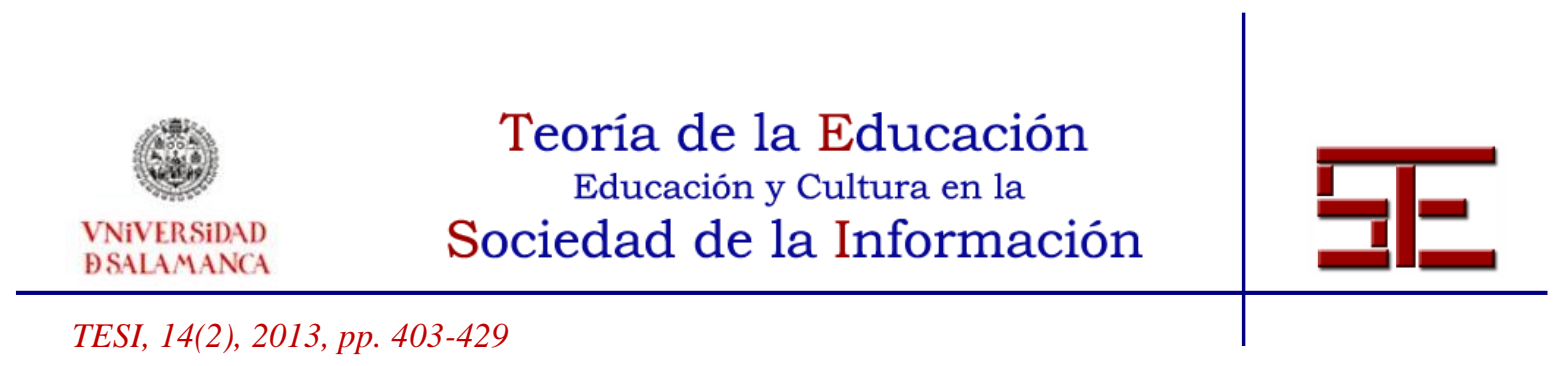

Como se puede observar, los profesores también tienen la opción de borrar los ejercicios, y generar automáticamente la información solicitada. Para poder identificar los verbos y los pronombres se ha utilizado OpenNLP (2012).

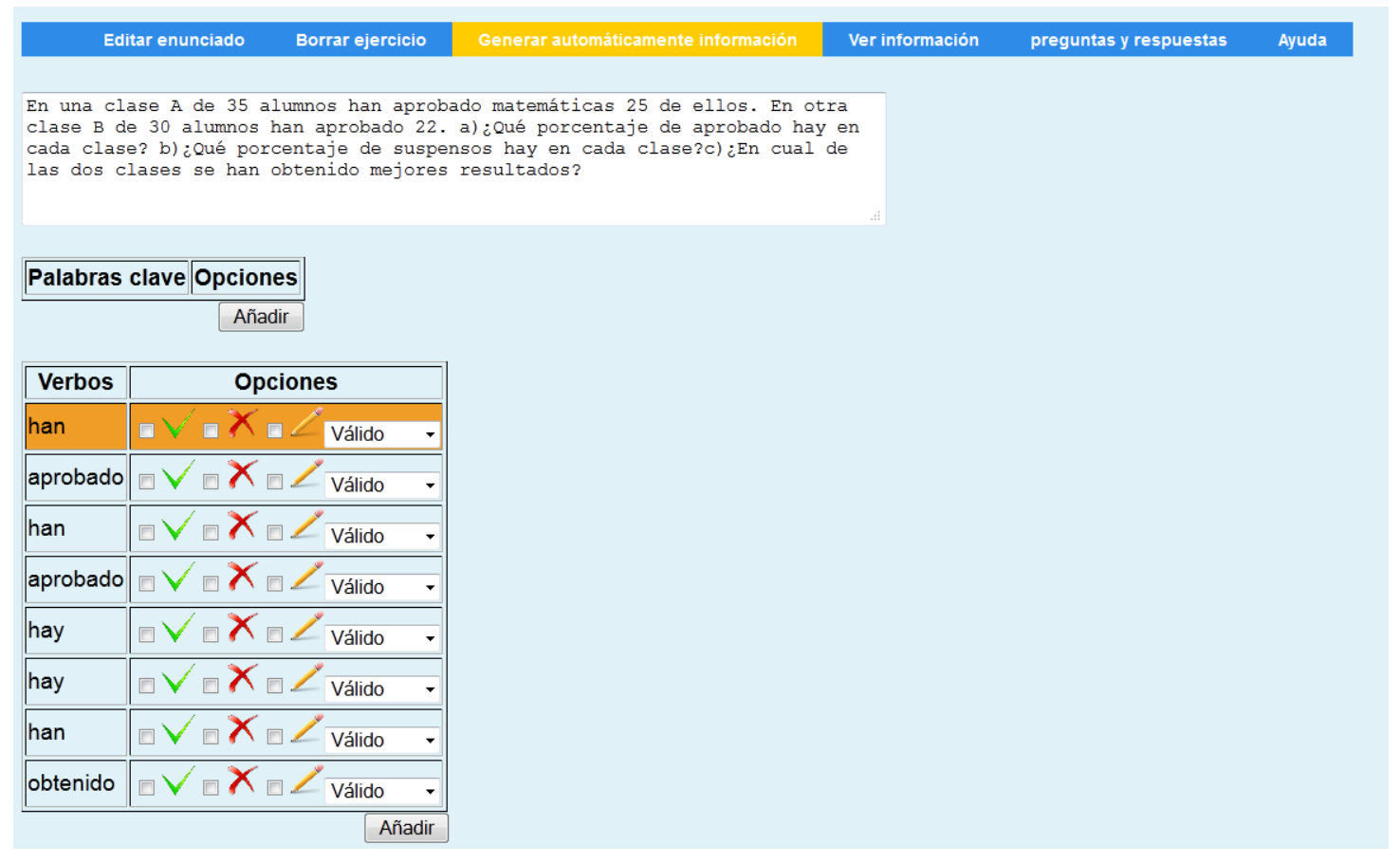

Figura 5. Interfaz de la operación editar enunciado

La generación es una operación supervisada por el profesor que es quien finalmente decide que la información generada es correcta. Además, el profesor puede visualizar toda la información generada de cada ejercicio y modificarla-borrarla en cualquier momento. También puede acceder al listado de preguntas y modificarlas, o borrar alguna ya existente.

Una vez validada la herramienta de gestión de preguntas y su información asociada con el profesor, se diseñó el algoritmo que guiará el diálogo entre Dr. Roland y el estudiante a partir de esta información. Para poder diseñar el algoritmo se tuvo en cuenta la opinión de los profesores que nos pidieron que se fuera ayudando a los estudiantes a llegar a la solución mediante ayuda progresiva y contextualizada en aquellos aspectos que les

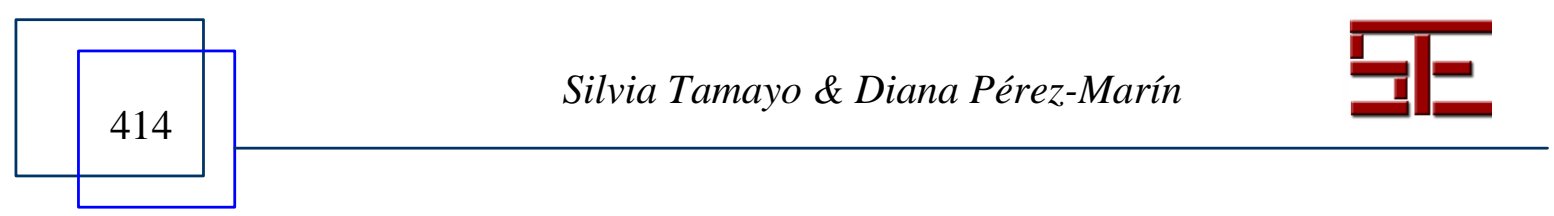




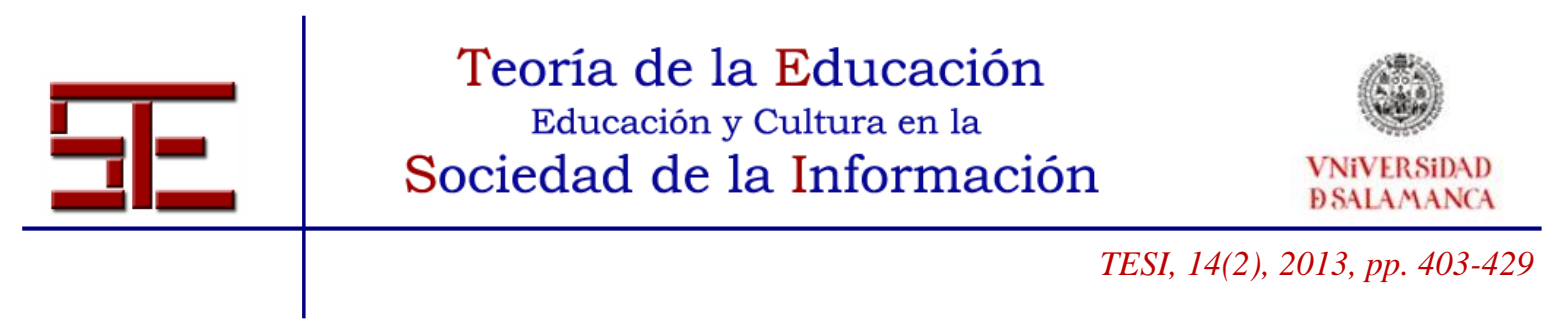

resultan más complicados, sin ofrecerles la respuesta directamente (Tamayo \& PérezMarín, 2012b).

Por último, se validó con los estudiantes y los profesores la interfaz de Dr. Roland para los estudiantes. Como se mostró en la Figura 1, y siguiendo lo que nos pidieron los profesores, es una interfaz simple y consistente. El diálogo del agente sigue el algoritmo RUANLP, que escoge cada ejercicio según su tipo (ecuaciones, reglas de tres, polinomios, etc.) y su nivel de dificultad, para adecuarse a cada estudiante, y va registrando los avances que va realizando cada estudiante y su puntuación.

Además, en cada ejercicio que el estudiante no sepa resolver por sí solo, va prestando más ayuda según la va requiriendo el usuario, teniendo como base los parámetros que inicialmente se seleccionaron (frase principal, verbos, pronombres y palabras clave) y fueron incluidos en el algoritmo, siendo ampliada dicha ayuda por nueva información que haya podido incluir el profesor en la parte del docente. De tal manera, que hasta que no se llegue el final de la ayuda recibida, o el estudiante no haya comprendido el ejercicio, el estudiante siempre podrá recibir más ayuda, hacer reintentos en la comprensión del ejercicio, verificar si lo que él ha entendido es lo que realmente le dicen, y en caso contrario, conocer la respuesta.

Si el ejercicio tiene varias preguntas, como se muestra en la Figura 6, se decidió que los estudiantes debían introducirlas en el orden que aparecían las preguntas y sólo acompañadas de las unidades del valor introducido como longitud, área, profundidad, etc. Además, también se adoptó el convenio de separar por comas las respuestas de una misma pregunta, y por puntos y coma cuando las respuestas son de preguntas distintas.

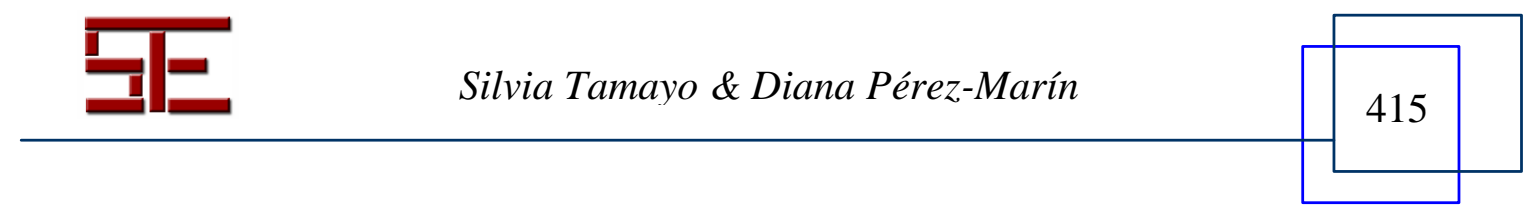



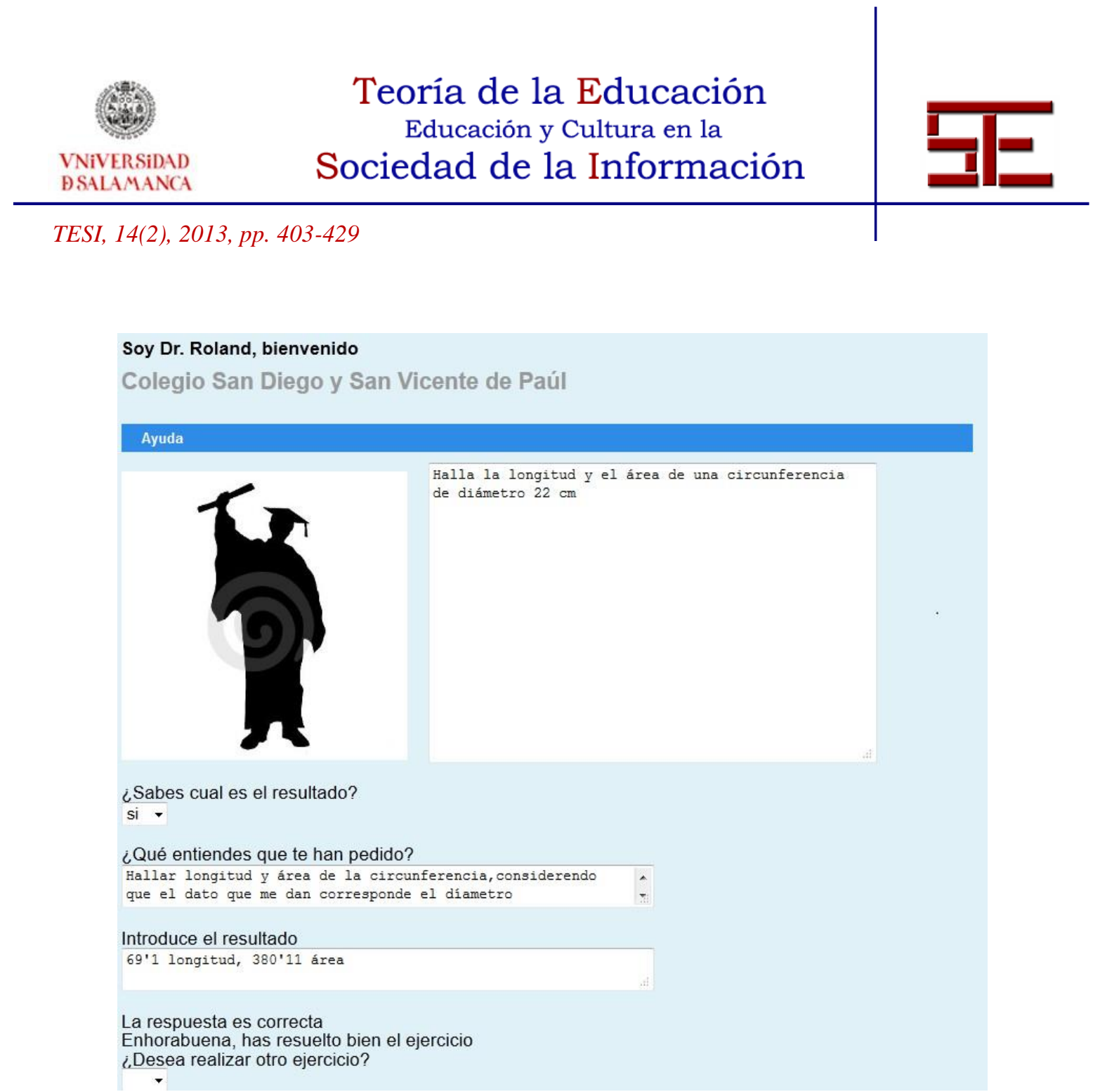

Figura 6. Interfaz de Dr. Roland para el estudiante

Otro aspecto que también se debatió es si sería adecuado/necesario incluir dentro de la aplicación algún tipo de calculadora o página donde realizar cuentas. Finalmente, puesto que en los exámenes de matemáticas en el colegio no se permite a esa edad el uso de calculadoras, no se incluyó, aunque los estudiantes acabaron usando en ocasiones la calculadora del propio sistema operativo o bien pidieron papel para realizar los cálculos.

Por último, se contempló la posibilidad de realizar actividades colaborativas pero puesto que el diálogo se había diseñado estudiante-agente, no se propuso, y se optó por un desarrollo web al que se pudiera acceder de manera individual (con su usuario y contraseña personal) desde cualquier ordenador conectado a Internet.

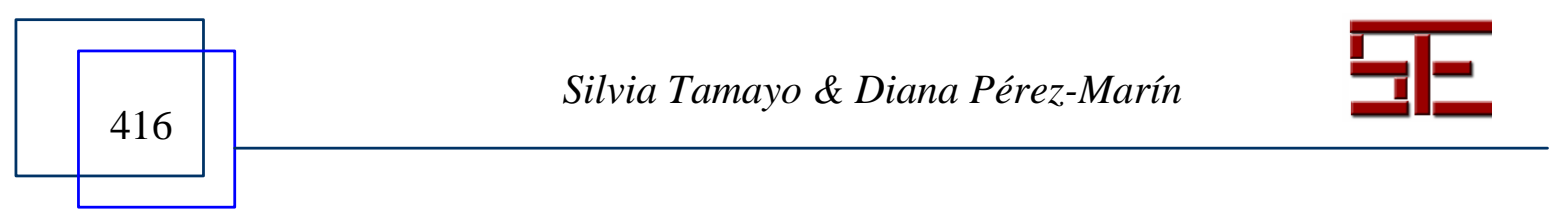




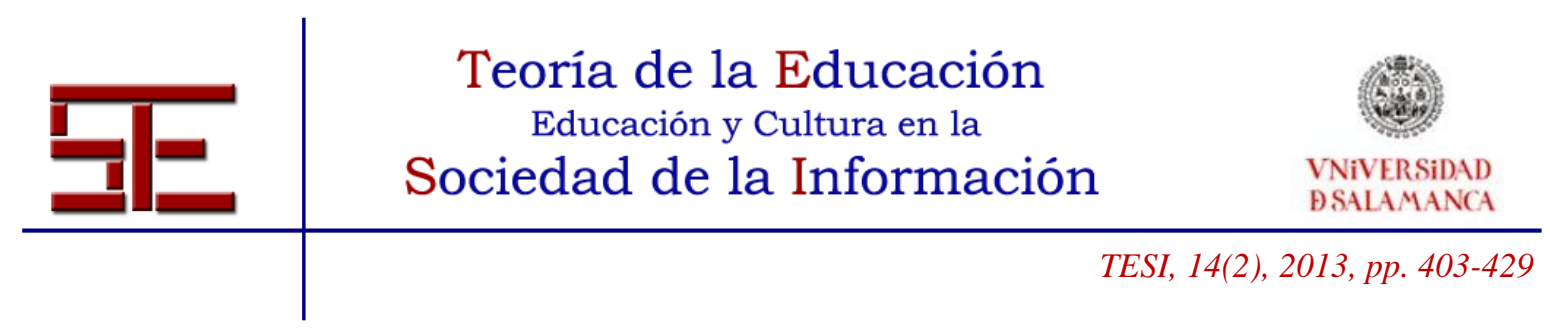

\section{EXPERIENCIA}

\subsection{Participantes $e$ instrumentos utilizados}

La muestra consistió en 38 niños de 12-13 años y 3 profesores de un colegio de Madrid que se ofrecieron voluntariamente a colaborar con nosotros tanto en el desarrollo del agente como en la experiencia de uso. Las características de los mismos, según se ha analizado mediante la realización de cuestionarios, se presentan a continuación:

- A todos los estudiantes les gustan los ordenadores, y tienen ordenador en casa.

- Todos los estudiantes excepto uno tiene Internet en casa.

- Todos los estudiantes tienen cuenta de correo electrónico.

- Sobre el permiso de sus padres para conectarse a Internet, un $48 \%$ de los estudiantes puede conectarse todos los días, y el resto de estudiantes puede algunas veces ( 1 estudiante indica que tiene prohibido navegar en casa).

- De los estudiantes que pueden conectarse a Internet todos los días, un $21 \%$ lo utiliza para alguna actividad académica, aunque la gran mayoría sólo lo usa a veces y solicitan poder usar el ordenador para estudiar no solo en casa sino también en clase en un $32,5 \%$ de los casos.

- Por último, respecto a su actitud ante las matemáticas, las opiniones que se recogen son muy diversas. La gran mayoría (51,9\% de los estudiantes) responde que le gustan a veces y que creen que son necesarias. De hecho, la opción mayoritaria escogida por un $65,1 \%$ de los estudiantes es que a veces los ejercicios son difíciles de comprender. Algunos estudiantes indican que: "depende del enunciado y de lo que sepas de matemáticas", "si, a mi me cuesta mucho entenderlos y tengo que volvérmelos a leer varias veces y aún así sigo sin comprenderlo", "hay que entenderlos pero son un poco difíciles", o "depende del problema que sea".

Otro instrumento que se ha utilizado son hojas de ejercicios en papel. Ambos grupos debían resolver los ejercicios en clase al principio y al final del experimento. Los ejercicios fueron seleccionados para cubrir distintas áreas de las matemáticas: números naturales y enteros, divisibilidad, fracciones, ecuaciones, sistema métrico decimal, proporcionalidad y porcentajes, estadística y probabilidad, y volúmenes. También se han clasificado para abarcar tres niveles de dificultad desde uno (más fácil) a tres (más difícil). No obstante, esta clasificación no se mostraba en los ejercicios por indicación de los profesores para no condicionar a los estudiantes y que pudieran pensar que los ejercicios más difíciles eran imposibles.

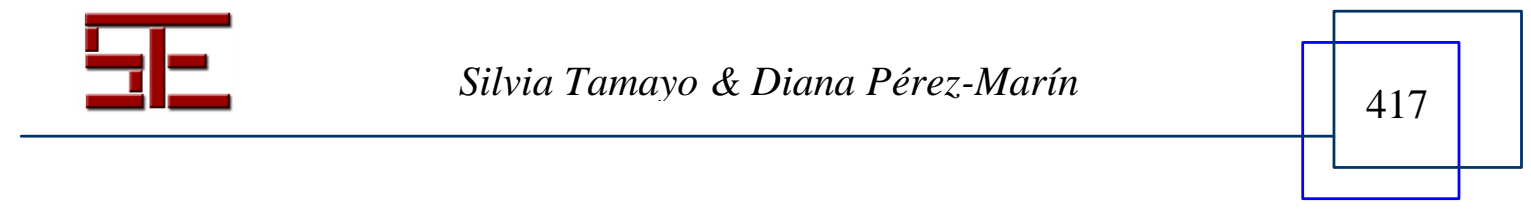




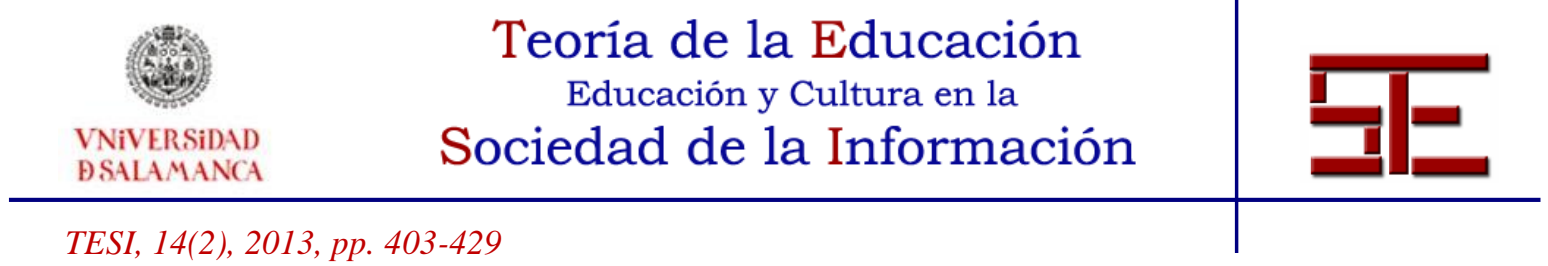

Los resultados de los ejercicios sirven para tener conocimiento, en el punto de partida, de la situación de la muestra en relación a los ejercicios de matemáticas, por lo que se realizan en ambos grupos antes del comienzo de la experiencia. Y la realización al final, sirve para contrastar los resultados obtenidos en ambos grupos, considerando que uno usa el agente (grupo test) y el otro no lo usa (grupo control).

Estos ejercicios son un subconjunto de una batería de ejercicios de matemáticas más amplia proporcionada por los profesores con 78 ejercicios de 8 tipos y 3 niveles adecuados a las características de la muestra, como material para el agente. Un resumen del número de ejercicios para cada nivel por tipo, puede encontrarse en la Tabla 1.

Tabla 1. Problemas de matemáticas proporcionados por los profesores

\begin{tabular}{|c|c|c|c|}
\hline Tipo & $\begin{array}{c}\text { \# de ejercicios } \\
\text { de nivel 1 }\end{array}$ & $\begin{array}{c}\text { \# de ejercicios } \\
\text { de nivel 2 }\end{array}$ & $\begin{array}{c}\text { \# de ejercicios } \\
\text { de nivel 3 }\end{array}$ \\
\hline Números naturales y enteros & 5 & 2 & 2 \\
\hline Divisibilidad & 0 & 1 & 1 \\
\hline Fracciones & 2 & 7 & 4 \\
\hline Ecuaciones & 1 & 2 & 8 \\
\hline Sistema métrico decimal & 0 & 5 & 3 \\
\hline Proporcionalidad y porcentajes & 11 & 6 & 0 \\
\hline Estadística y probabilidad & 4 & 3 & 1 \\
\hline Medidas y volúmenes & 3 & 4 & \\
\hline
\end{tabular}

Para la realización de las reuniones de DCU con los docentes, fundamentales en el proceso, los lugares elegidos fueron aquellos que se adaptaban mejor a la situación en cada momento. No obstante, cabe destacar que el lugar más habitual fue una cafetería, con motivo de la necesidad adaptarse a la disponibilidad de tiempo de los docentes en su horario de trabajo habitual, y con los horarios de trabajo del resto de participantes. Durante las reuniones, los docentes, para responder a preguntas que se les realizaba sobre la muestra y otros aspectos relacionados con ella, así como hacer consultas, usaban una tableta donde tenían disponible toda la información relativa a la muestra.

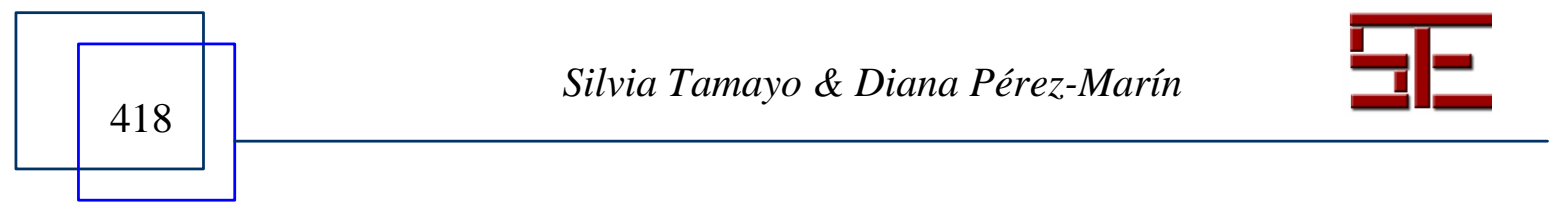




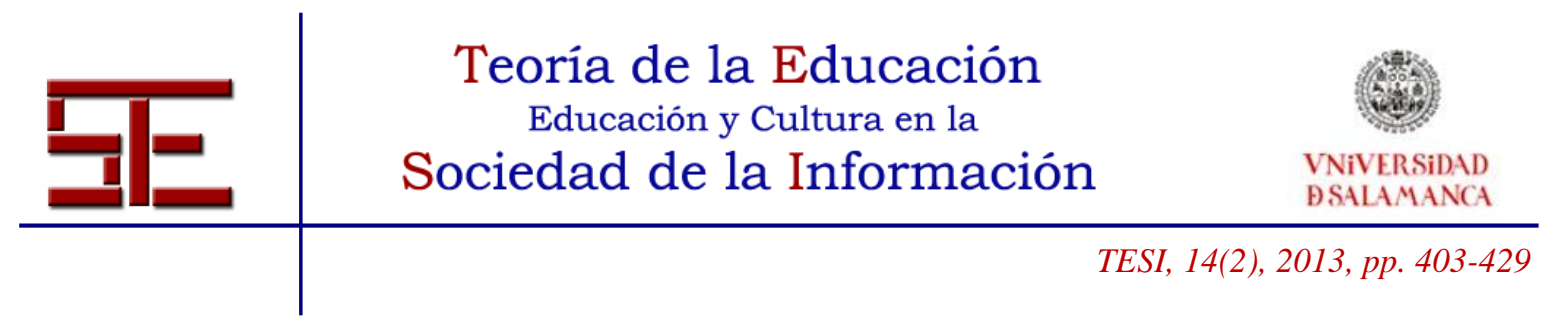

También se emplearon, en las primeras fases, prototipos en papel, donde se les mostraba a los docentes, una primera aproximación del agente, tanto la interfaz del profesor como la interfaz del alumno. Posteriormente, se fueron mostrando prototipos horizontales de baja fidelidad web, para ir validando los prototipos del agente a medida que iba evolucionando su desarrollo.

Para el uso del agente por parte de los niños, se ha dispuesto de dos aulas en el colegio al que pertenece la muestra. Un aula de teoría, para la realización de los cuestionarios y de los ejercicios en papel, y un aula de informática, para el uso del agente por los niños, con un ordenador para cada estudiante. Además, se ha contado con un servidor en funcionamiento continuo en la Universidad Rey Juan Carlos en el que se ha alojado la aplicación.

\subsection{Diseño y desarrollo de la experiencia}

Se realizó un diseño de la experiencia según una estructura de grupo control-test con prepost test. La Figura 7 muestra gráficamente el desarrollo de la experiencia. El 24 de abril fue la primera vez que se fue al colegio y se pidió a los 38 estudiantes que completaran el cuestionario inicial para recabar información sobre la muestra, y calificar su pre-test ( 8 ejercicios de 4 niveles de dificultad).

También se validó la interfaz de Dr. Roland con los 19 estudiantes (grupo test) que usaron el agente. El grupo control fue constituido con otros 19 estudiantes que no usaron el agente. Estos estudiantes al pertenecer a grupos diferentes en el colegio no hablaron entre ellos. Además, como el agente era on-line, se pidió a los estudiantes del grupo test que siguieran usando Dr. Roland en casa. También se les pidió que participaran por correo en un concurso para seleccionar la cara de Dr. Roland.

Sin embargo, no se recibió ningún correo y tampoco se registró ningún uso de Dr. Roland. Por lo tanto, se solicitó a los profesores volver a clase el 3 de mayo de 2012 para poder recoger más información sobre el uso del sistema, y finalmente el 22 de mayo se pidió a los estudiantes completar el post-test, de nuevo 8 ejercicios de 4 niveles de dificultad tanto en el grupo control como en el test para estudiar si había diferencias significativas entre ambos grupos y sus opiniones al respecto.

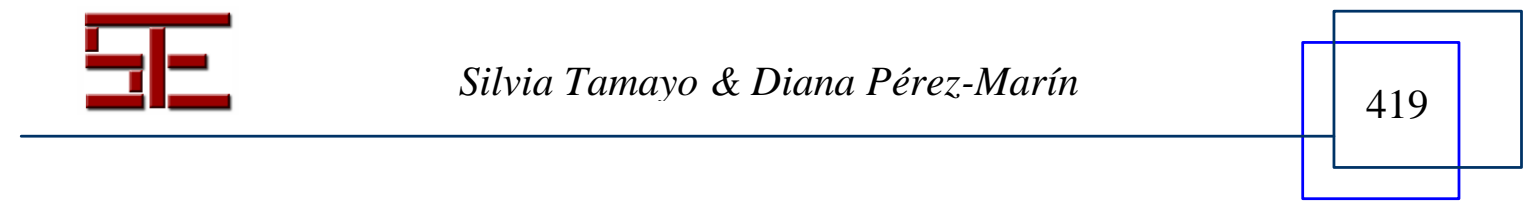



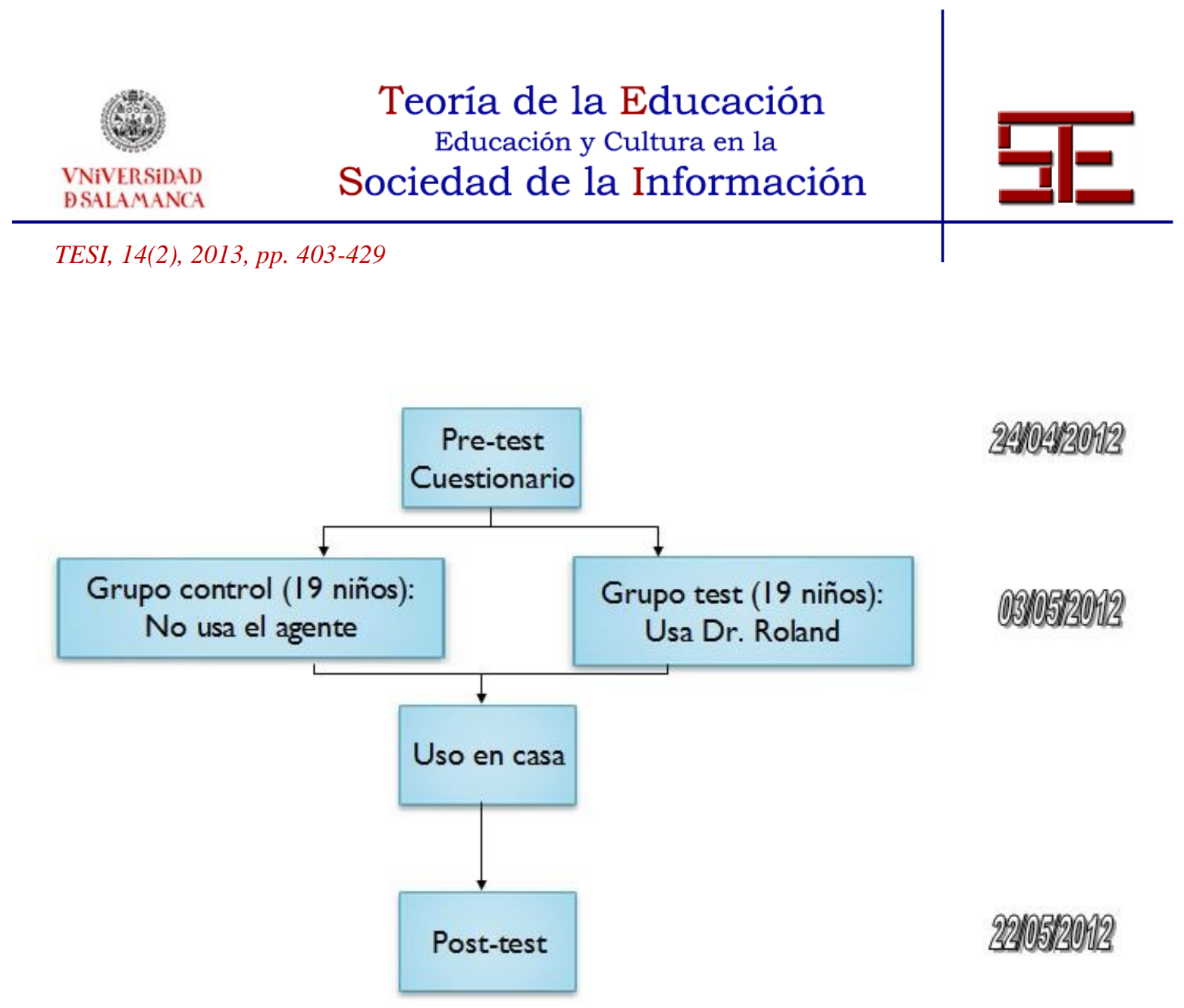

29048012

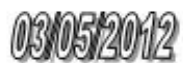

Figura 7. Diseño de la experiencia

\subsection{Resultados}

Los niños no tuvieron ningún problema interactuando con la interfaz, y supieron manejar Dr. Roland desde el principio. Sin embargo, se registraron dificultades a la hora de seguir el formato de entrada cuando el ejercicio tenía varios resultados para que fuera evaluado correctamente por el sistema.

Todos los estudiantes estuvieron atentos durante la clase, y se involucraron en la realización de los ejercicios, preguntando dudas y mostrando un alto nivel de interés. En relación a los comentarios que realizaban durante el uso del agente, los principales se centraban en:

- Cálculos que no sabían realizar.

- El significado de palabras que desconocían.

- Comparaciones entre ellos como: “yo ese ejercicio ya lo resolví, aunque no lo hice

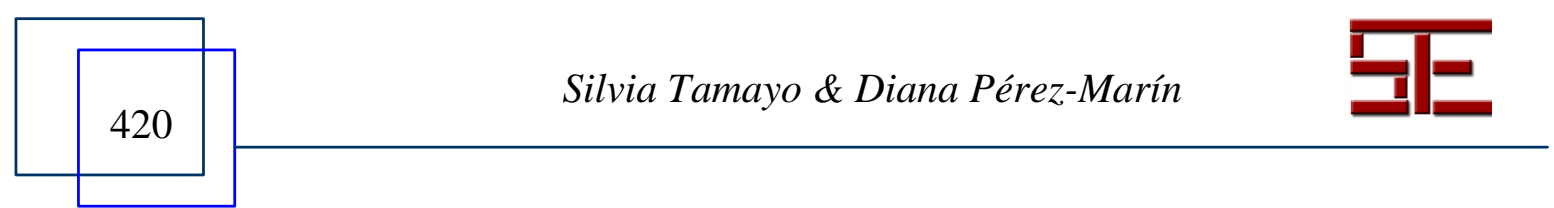




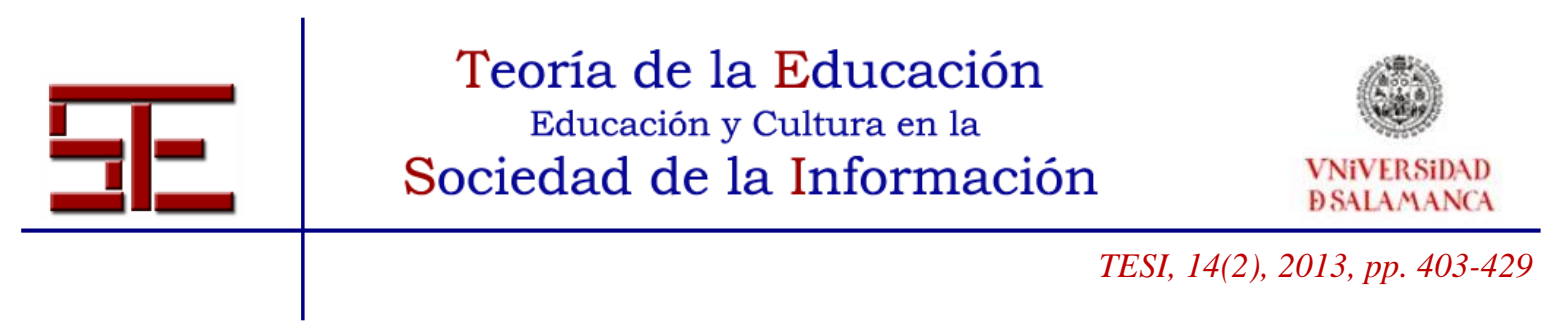

bien", "yo ya pasé por ese ejercicio", "he realizado x ejercicios", "he tenido bien $x$ ejercicios", "me he equivocado en $x$ ejercicios", "voy $x$ ejercicios más adelantado que tú", "ya hice el ejercicio, así que si tienes dudas en los cálculos pregúntame si quieres", etc.

Del análisis de los comentarios, se quiere destacar la actitud positiva y participativa de los niños frente al agente y sus compañeros. Así como la motivación, muy superior a cuando se realizaron los ejercicios en papel. Respecto a los resultados numéricos, el análisis cuantitativo revela que en una escala de 0 (nota más baja) - 8 (nota más alta), los estudiantes del grupo control alcanzaron la nota máxima de 3 en el pre-test, y un promedio de 1,53 (desviación típica=1,1). En el caso del grupo test, siguiendo la misma escala, en el pre-test de nuevo la nota máxima también es un 3, y el promedio es de 1,78 (desviación típica=0,94). Por lo tanto, todos los estudiantes suspenden.

Cuando se pide que completen el post-test a finales de mayo, en el caso del grupo control 4 estudiantes aprueban (un $21 \%$ del grupo) y la nota media asciende ligeramente a 2,1 (desviación típica=1,4) que sigue siendo de suspenso aunque ya recogiendo algunos aprobados. Lo que era de esperar puesto que han continuado estudiando matemáticas. En el caso del grupo test, que utilizaron el agente, uno de los estudiantes es capaz de alcanzar un 5, y 2 estudiantes aprueban (un $10 \%$ del grupo) y la nota media asciende ligeramente a 2,2 (desviación típica=1,18). Por lo tanto, no se puede recoger evidencia significativa de que el uso del agente haya supuesto una mejora en la capacidad de resolución de los ejercicios. Esto puede deberse al limitado tiempo de uso del agente a las tres visitas al colegio.

Para completar este análisis global de la capacidad de resolución de ejercicios, se quiere estudiar, a continuación, la variación en el nivel de comprensión de los ejercicios. Para ello, se calificaron los ejercicios realizados en la primera y última sesión con el agente según cómo los estudiantes habían sido capaces de comprender cada ejercicio y había quedado registrado en el sistema. De esta forma, se podía medir la variación en el nivel de comprensión de los ejercicios también. En esta ocasión, el estudio se realiza para cada uno de los estudiantes y tipos de ejercicios, ya que cuando la mayoría de los estudiantes no ha tenido ocasión de abordar ejercicios de algún tipo con el agente no se puede esperar mejoras ni en la comprensión ni en la resolución.

La Figura 8 muestra el contraste de las variables comprensión y resolución de los ejercicios de ecuaciones; la Figura 9 para los ejercicios relacionados con el sistema métrico decimal; la Figura 10 para los ejercicios fracciones; la Figura 11 para los

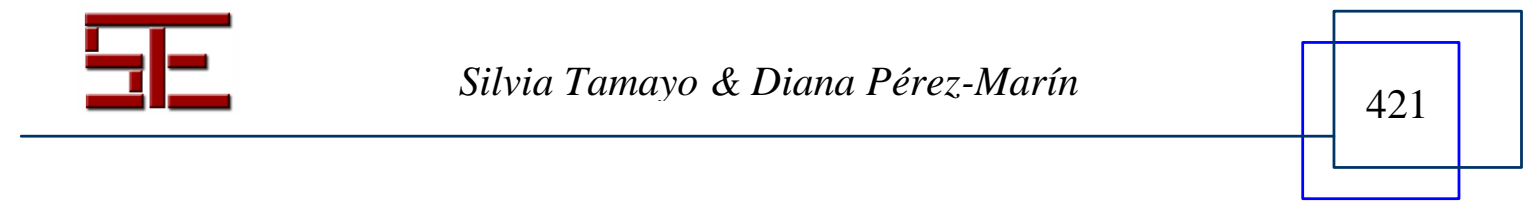




\begin{tabular}{c} 
Teoría de la Educación \\
Educación y Cultura en la \\
$\begin{array}{c}\text { VNIVERSiDAD } \\
\text { DSALAMANCA }\end{array}$ \\
\hline TESI, 14(2), 2013, pp. 403-429
\end{tabular}

ejercicios proporcionalidad y porcentaje; la Figura 12 para los ejercicios medidas y volúmenes; y la Figura 13 para los ejercicios estadística y probabilidad. En todos los casos, el eje horizontal representa los usuarios y el eje vertical la variación (en azul en comprensión y en rojo en resolución).

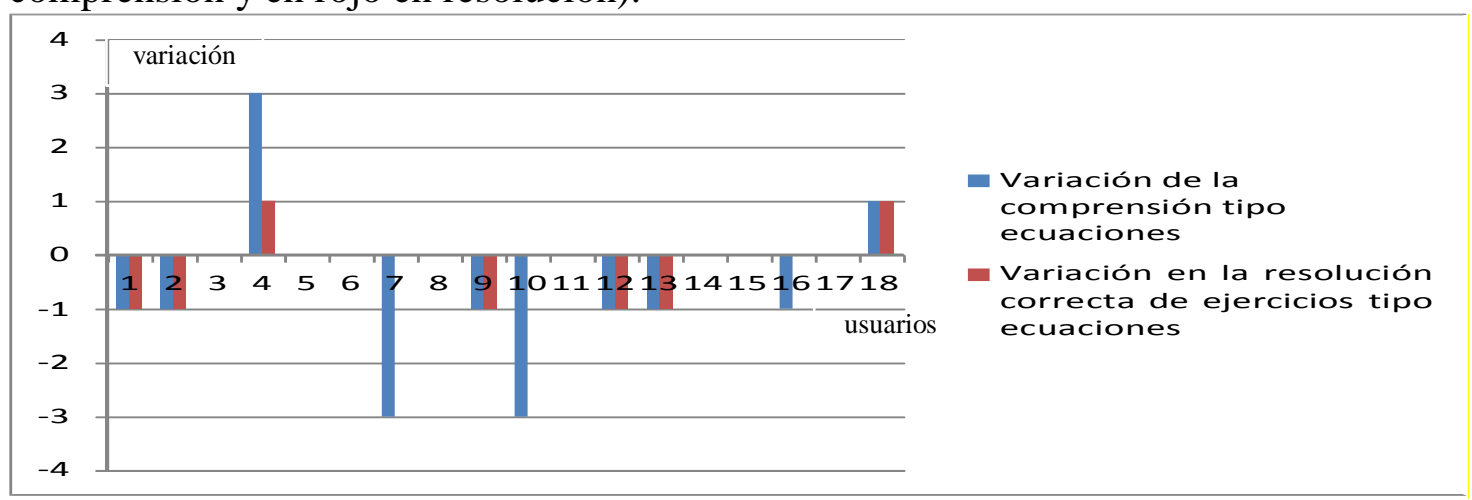

Figura 8. Variables comprensión y resolución de ejercicios de ecuaciones

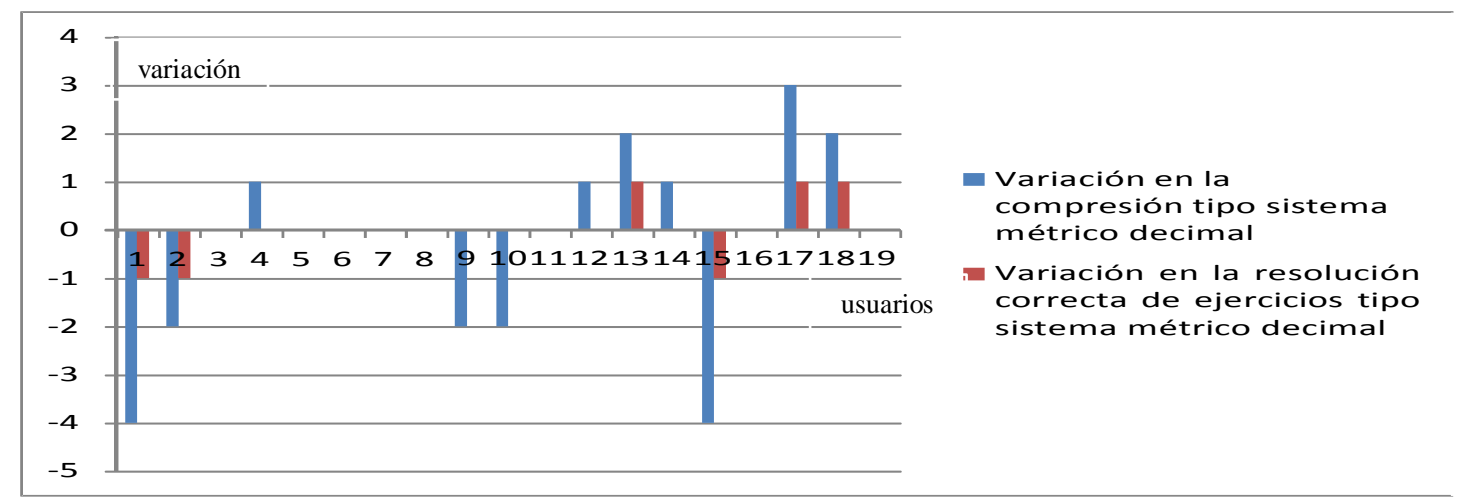

Figura 9. Variables comprensión y resolución de ejercicios de sistema métrico decimal

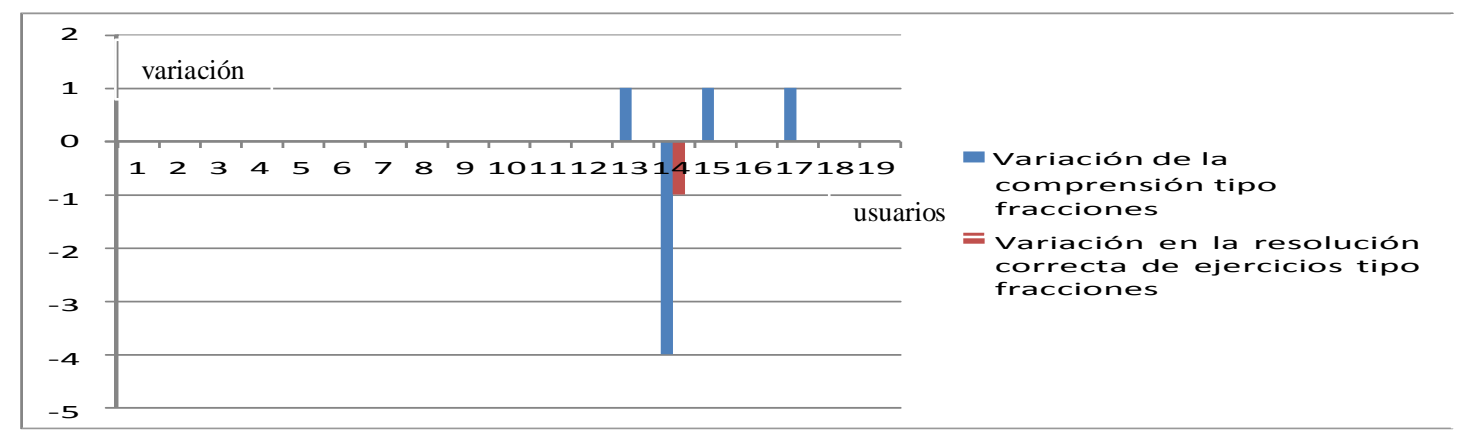

Figura 10. Variables comprensión y resolución de ejercicios de fracciones

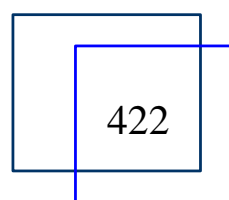

Silvia Tamayo \& Diana Pérez-Marín 

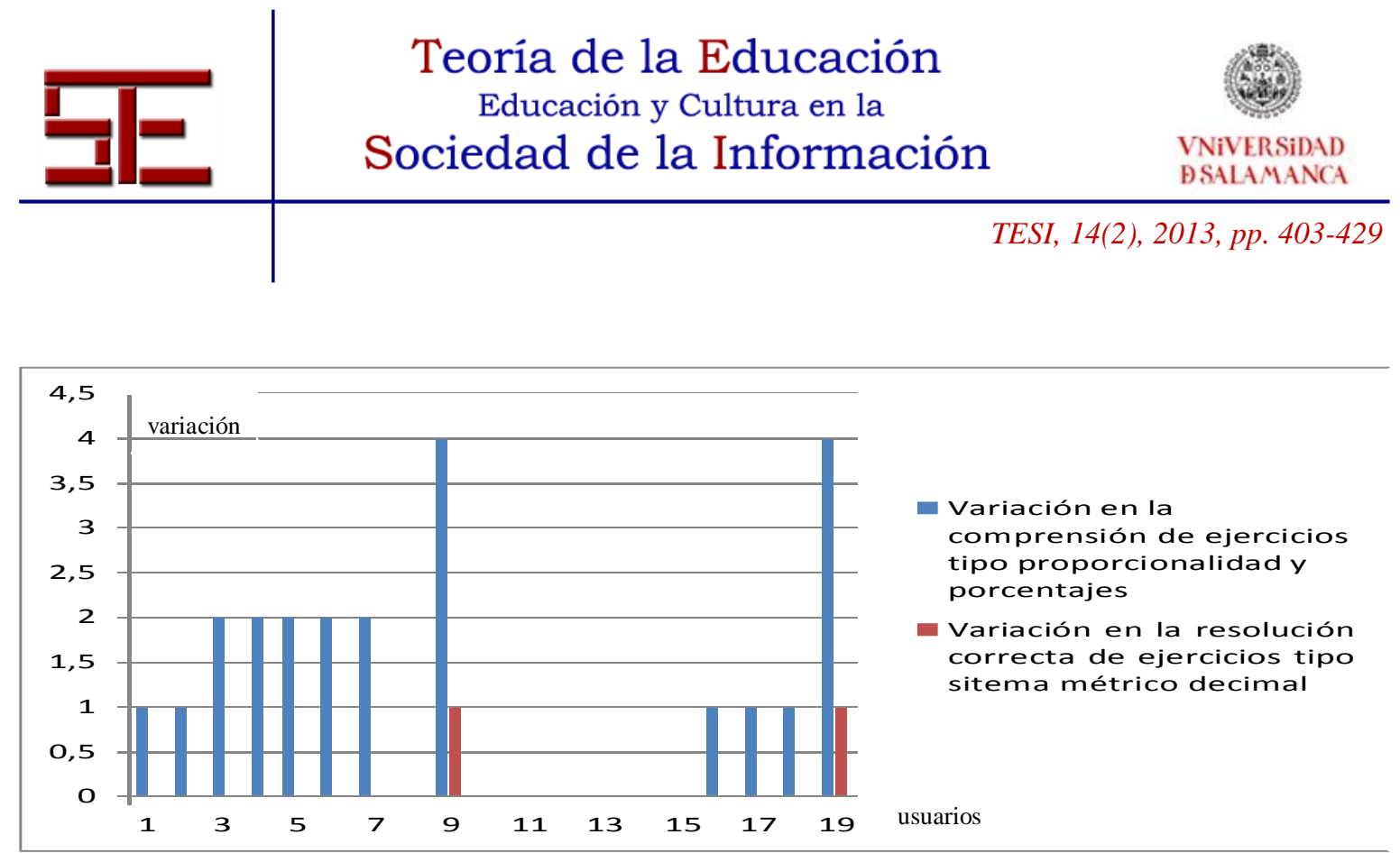

Figura 11. Variables comprensión y resolución de ejercicios de proporcionalidad y porcentaje

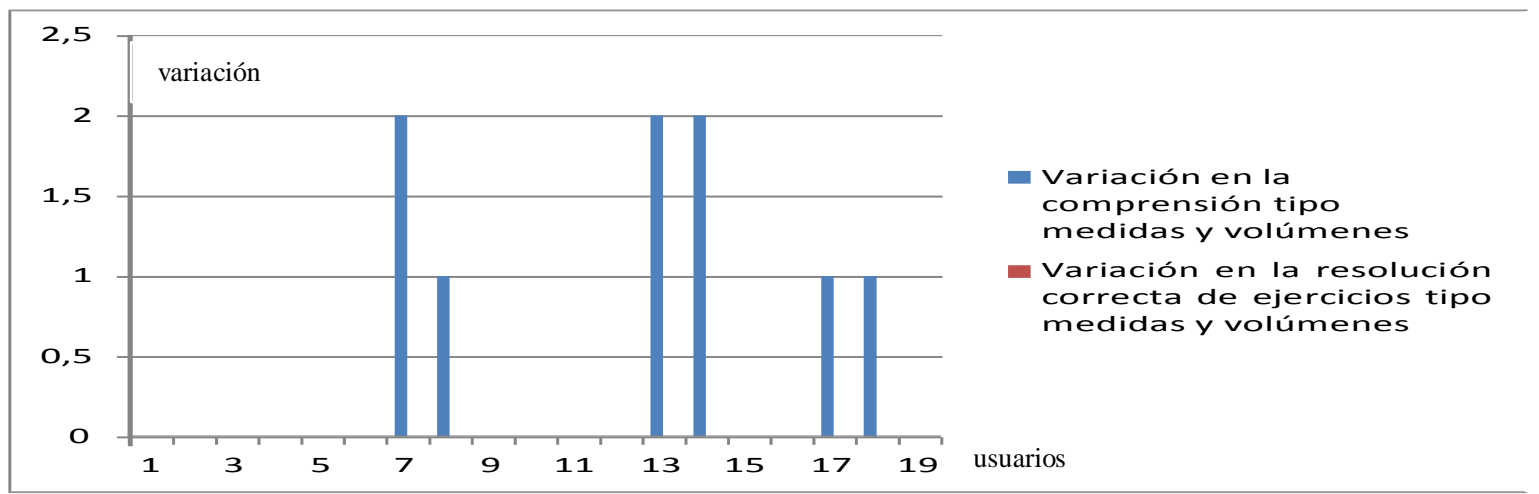

Figura 12. Variables comprensión y resolución de ejercicios de medidas y volúmenes

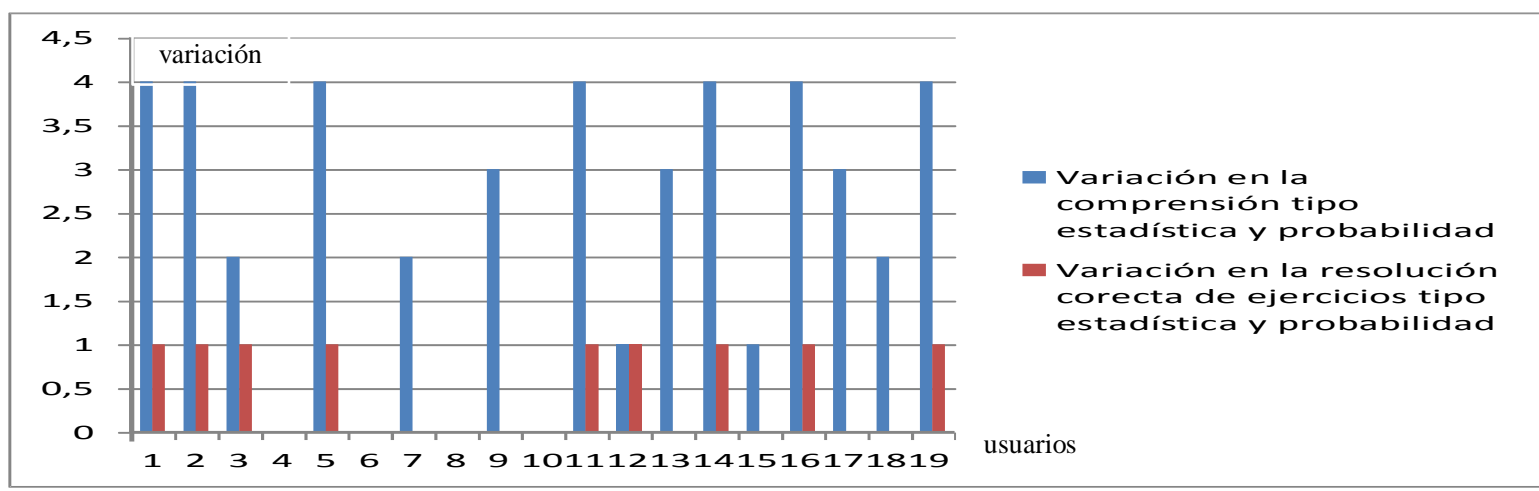

Figura 13. Variables comprensión y resolución de ejercicios de estadística y probabilidad

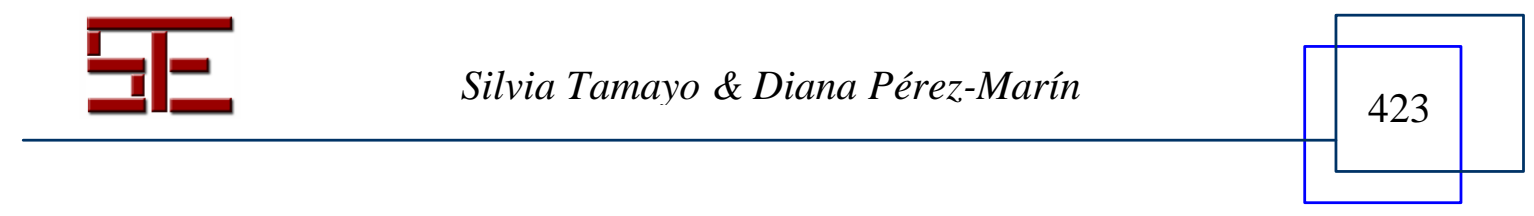




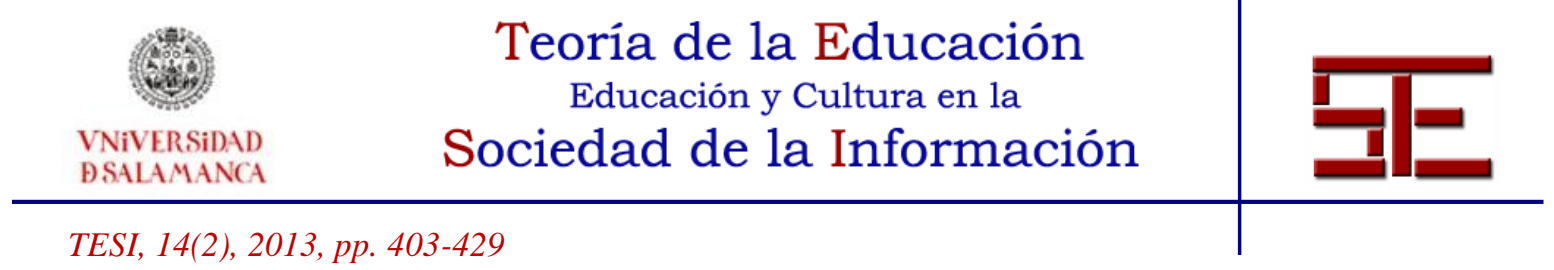

A nivel global, como se puede apreciar en las gráficas, hay casos que registran variación en la comprensión y sin embargo no se registra cambio en la capacidad de resolución, lo que invalidaría H2. Esto es, se puede mejorar la capacidad de comprensión sin que esto signifique una mejora en la capacidad de resolución de los ejercicios. Así, por ejemplo, en el caso de los ejercicios del sistema métrico decimal 6 estudiantes mejoran su capacidad de comprensión, y sólo 3 su capacidad de resolución y muy levemente.

De forma similar, en el caso de los ejercicios de fracciones, 3 estudiantes mejoran su capacidad de comprensión y ninguno la capacidad de resolución. Para los ejercicios de medidas y volúmenes sólo se registran mejoras en la capacidad de comprensión, y en el resto de ejercicios es notable cómo se registran mejoras en la capacidad de comprensión pero no en la resolución.

\section{CONCLUSIONES}

Los Agentes Conversacionales Pedagógicos se pueden utilizar en clase y dar soporte a distintos dominios, como por ejemplo, se ha probado en el caso de resolver problemas de matemáticas. En particular, se ha podido desarrollar un agente que gestiona el diálogo con los estudiantes para guiarles en la comprensión de los ejercicios con la ayuda de 3 profesores y 1 informático como desarrollador de la aplicación. Esto significa que no es necesaria una gran inversión tecnológica ni presupuestaria.

Además, las herramientas imprescindibles suelen estar al alcance de la mayoría de los colegios hoy en día, como una sala con ordenadores, y un ordenador que será el servidor donde se instale la aplicación (en este caso al ser web, si no fuese on-line no sería necesario). De hecho, no se han encontrado beneficios en realizar la aplicación on-line, puesto que los estudiantes han seguido usándola desde los ordenadores en clase, y al no involucrar a los padres en el estudio no se ha conseguido fomentar el uso del agente en casa. Esto ha tenido también como consecuencia que el tiempo de uso del agente ha sido limitado y no se han podido encontrar resultados significativos.

Se han registrado variaciones positivas en el caso de la comprensión de algunos ejercicios, a diferencia de su capacidad de resolución que no ha tenido una mejoría observable. De hecho, las diferencias entre el grupo control que no usó el agente y el grupo test que usó el agente no son significativas por lo que cualquier mejoría puede no ser atribuible al uso del agente.

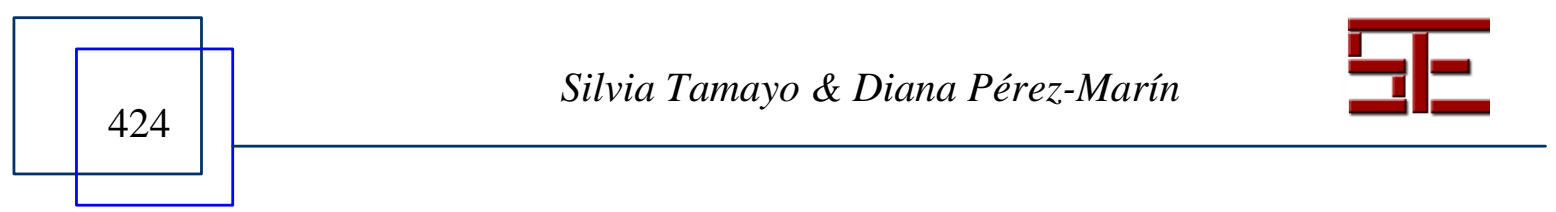




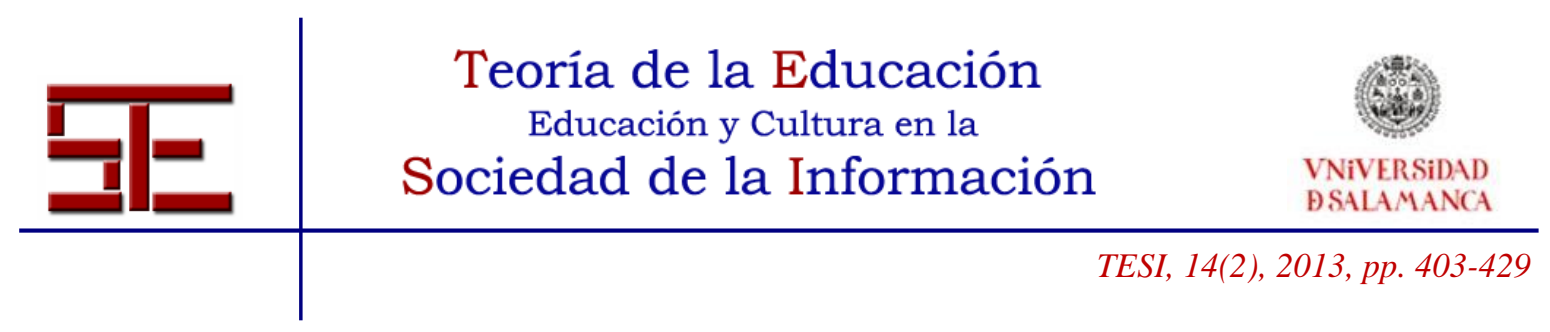

También se observa que los estudiantes han disfrutado del uso del agente en clase, y han estado atentos durante su interacción con el sistema. No han encontrado dificultades en su manejo. Los estudiantes han entendido correctamente el objetivo del sistema y tan solo han cometido errores a la hora de introducir los resultados cuando eran complejos.

Dos comentarios que expresan muy bien lo analizado en este sentido son según los profesores:

"proyectos como el realizado, y embarcarse en otros de similares es importante, de gran utilidad para los alumnos, y además son métodos de enseñanza alternativos a los tradicionales, estando más relacionados con la nueva realidad de la sociedad y de cara a la importancia de las nuevas tecnologías"

y para los estudiantes:

"la utilidad de estos agentes en los procesos de aprendizaje, como método alternativo de enseñanza y más relacionado con sus costumbres, que es el uso de tecnologías prácticamente a diario. Destacando la mayor motivación y actitud participativa, así como la importancia que conceden a que el agente les ayude".

Como resultado de esta experiencia se quiere animar a otros profesores, estudiantes y profesionales que quieran innovar en las tecnologías educativas e incorporar Agentes Conversacionales Pedagógicos en sus clases. Se pueden integrar y combinar con otros métodos tradicionales de enseñanza como se hizo en la experiencia en el curso 2011/2012 con los 19 estudiantes del grupo test que fueron a clase con el ordenador algunos días y otros días siguieron sus clases tradicionales.

También se quiere proporcionar unas guías sobre cómo desarrollar agentes teniendo en cuenta la opinión de los estudiantes y profesores desde el principio, que son las siguientes:

- Identificar un problema real sin resolver con la ayuda de los usuarios. Esto permite que les interese más el desarrollo puesto que consideran que es útil y necesario.

- Estudiar cómo puede abordarse el problema a resolver identificando qué roles de usuarios ayudarán en cada caso, y planificando un calendario de reuniones para cada uno de los roles.

- Realizar una búsqueda de una muestra de cada rol identificando que pueda colaborar en el desarrollo informático según el calendario previsto.

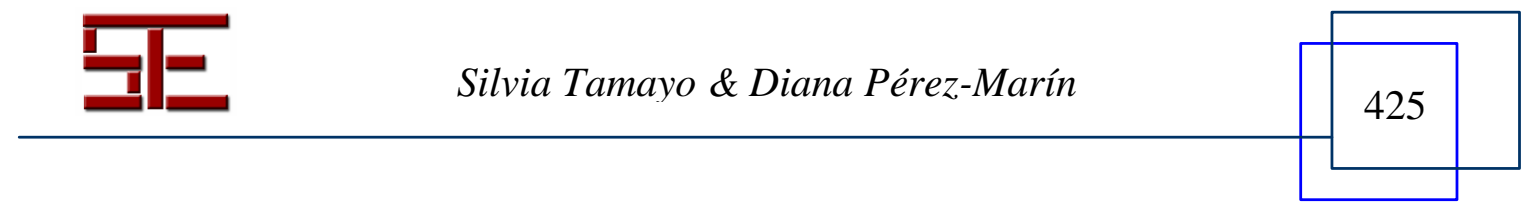




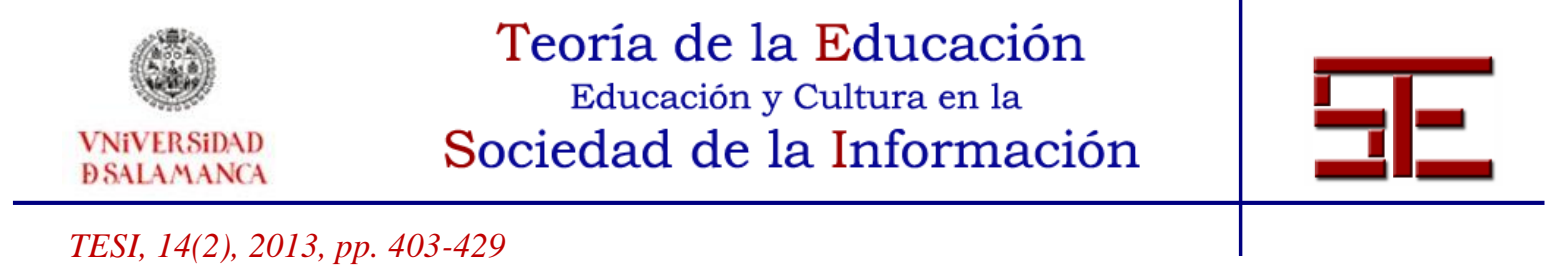

- Organizar y llevar a cabo una reunión inicial con ellos, escuchar su opinión y recabar la información necesaria para la creación de un primer prototipo informático a partir de un prototipo en papel que se les propone. Se considera fundamental en este punto proporcionar algún tipo de soporte papel para que los usuarios puedan modificarlo sin necesidad de conocimientos informáticos y con libertad para expresarse.

- Organizar y llevar a cabo una segunda reunión una vez terminado el primer prototipo y sin avanzar más en la codificación para validarlo con los usuarios y confirmar que el algoritmo principal del sistema corresponde a lo solicitado por los usuarios.

- Aceptar las posibles modificaciones que propongan los usuarios para que vean cómo se atienden sus sugerencias, y plantear nuevos cambios hasta llegar a una versión aceptada por los usuarios.

- Organizar la fase de pruebas de aceptación con otros usuarios para tener la oportunidad de extraer información a partir de la observación directa de uso del sistema, cuestionarios, logs y entrevistas personales si la muestra lo permite.

- Analizar los resultados obtenidos, establecer conclusiones y establecer a partir de estas conclusiones los siguientes pasos a dar con la ayuda también de los usuarios.

Por último, se quiere también ofrecer recomendaciones más generales para cualquier persona interesada en usar Agentes Conversacionales Pedagógicos:

- Incluir en la experiencia no solo a profesores y estudiantes, sino también a padres para que los niños usen el agente en casa.

- No preguntar directamente a los niños sino observar su uso del sistema.

- Diseñar y validar cada uno de los pasos sin suposiciones.

- Permitir a los niños el uso de lápiz y papel.

- Incluir en el sistema ayudas a la resolución de fórmulas.

- Incluir ejemplos para explicar el uso del sistema.

- Mantener la interfaz tipo Messenger para ofrecer mayor flexibilidad a la entrada de los datos.

- Probar el uso de agentes con tabletas y/o smartphones.

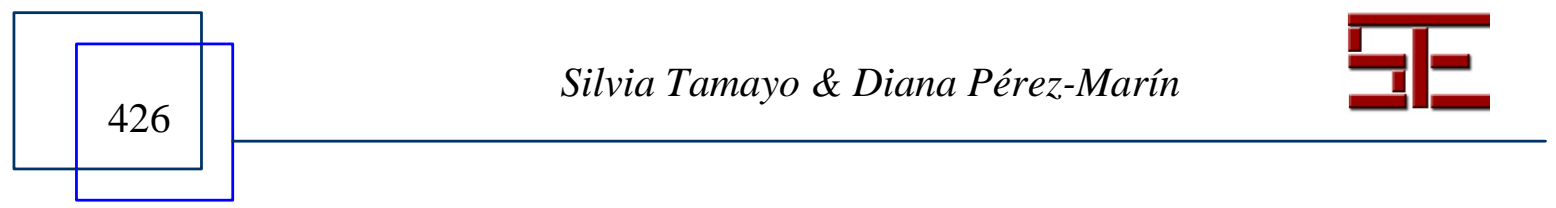




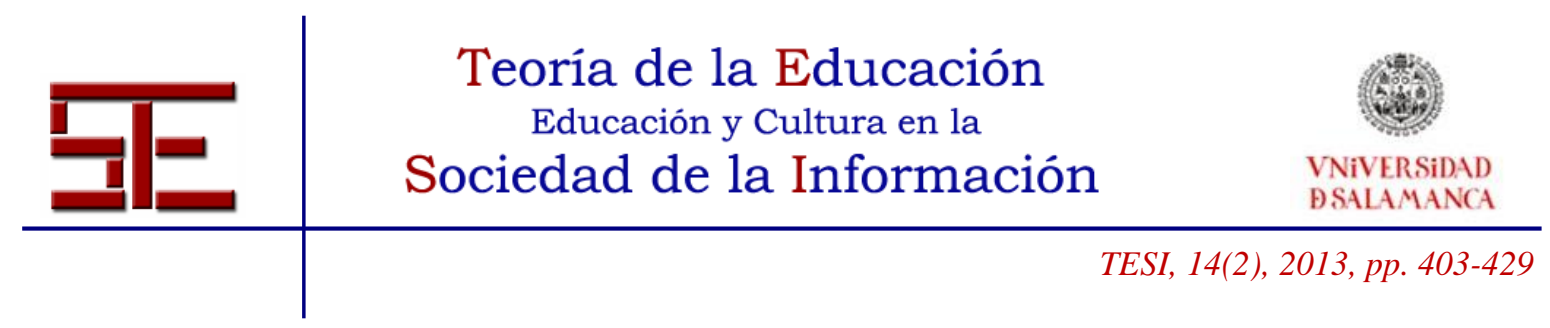

- Incluir posibilidades colaborativas (los niños acaban trabajando en grupo).

- Mantener el sistema de ajuste de niveles de dificultad y añadir un sistema de premios por cada nivel que el niño supere.

\section{5.- BIBLIOGRAFÍA}

Baroody, A. (1987). Children's mathematical thinking: a developmental framework for preschool, primary, and special education teachers, Teachers College Press.

Carletta, J., Isard, A., Isard, S., Kowtko, J., Doherty-Sneddon, G., Anderson, A. (1996). HCRC dialogue structure coding manual, Human Communication Research Centre.

Cassell, J. (2000). Embodied conversational interface agents. Communications of the ACM, 43(4), 70-78.

Chase, C., Chin, D., Oppezzo, M., \& Schwartz, D. (2009). Teachable agents and the protégé effect: Increasing the effort towards learning, Journal of Science Education and Technology 18, 334-352.

DAMSL. http://annotation.exmaralda.org/index.php/DAMSL. 18-05-2012.

Dussel, I., Quevedo, L.A., \& Santillana, F. (2011). Educación y nuevas tecnologías: los desafíos pedagógicos ante el mundo digital, Fundación Santillana.

Ginsburg, H. (1977). Children's arithmetic: The learning process. Van Nostrand Reinhold.

Graesser, A., Penumatsa, P., Ventura, M., Cai, Z., \& and Hu, X. (2007). Using LSA in AutoTutor: Learning through mixed initiative dialogue in natural language. In $\mathrm{T}$. Landauer, D. McNamara, S. Dennis, and W. Kintsch (Eds.), Handbook of Latent Semantic Analysis, Mahwah, NJ: Erlbaum, 243-262.

Graesser, A., Person, N., \& Harter, D. (2001). Teaching tactics and dialog in AutoTutor, International Journal of Artificial Intelligence in Education 12(3), 23-29.

Hays, M., Lane, C., Auerbach, D., Core, M., Gomboc, D. \& Rosenberg, M. (2009). Feedback Specificity and the Learning of Intercultural Communication Skills, Artificial Intelligence in Education.

Johnson, W., Rickel, J., \& Lester, J. (2000). Animated Pedagogical Agents: Face-to-Face Interaction in Interactive Learning Environments, Journal of Artificial Intelligence in Education 11, 47-78.

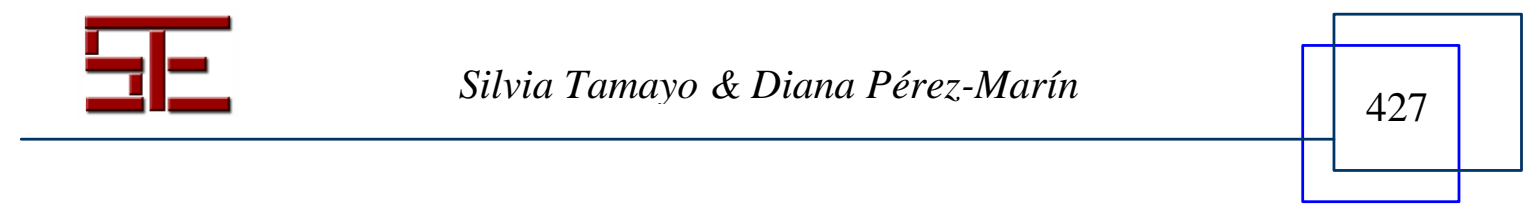




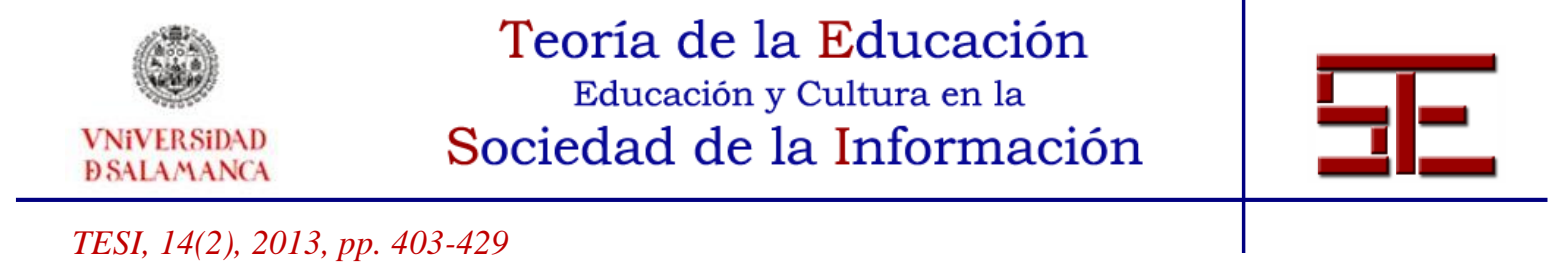

Kerly, A., Ellis, R. \& Bull, S. (2008). Conversational Agents in E-Learning CALMsystem: A Conversational Agent for Learner Modelling. Knowledge Based Systems, 21(3), 238-246.

Lester, J., Converse, S., Kahler, S., Barlow, S., Stone, B. \& Bhogal, R. (1997). The persona effect: affective impact of animated pedagogical agents, Proceedings of the SIGCHI conference on Human factors in computing systems.

Mas, A. (2005). Agentes software y sistemas multiagente: conceptos, arquitecturas y aplicaciones. Prentice Hall.

Nielsen, J. (1993). Usability Engineering. Morgan Kaufmann.

OpenNLP. http://opennlp.apache.org/. 18-05-2012.

Pérez-Marín, D. \& Pascual-Nieto, I. (2011). Conversational Agents and Natural Language Interaction: Techniques and Effective Practices. IGI Global.

Pérez-Marín, D. (2007). Adaptive Computer Assisted Assessment of free-text students' answers: an approach to automatically generate students' conceptual models. Universidad Autónoma de Madrid.

Reategui, E., Polonia, E. \& Roland, L. (2007). The role of animated pedagogical agents in scenario-based language e-learning: a case study. Conference ICL.

Ryokai, K., Vaucelle, C., \& Cassell, J. (2003). Virtual peers as partners in storytelling and literacy learning, Journal of computer assisted learning, 19(2), 195-208.

Tamayo, S. \& Pérez-Marín, D. (2012a). Towards the Development of a Reading Comprehension Conversational Agent for Children applying User-Centered Design Techniques for Teachers and Students. In: M. Kravcik, O.C. Santos, J.G. Boticario, D. Pérez-Marín (Eds.) Proceedings of the 2nd International Workshop on Personalization Approaches in Learning Environments (PALE), CEUR Workshop Proceedings, 23-28.

Tamayo, S. \& Pérez-Marín, D. (2012b). Propuesta de Agente para la Comprensión Lectora. Aplicado a la Resolución de Ejercicios de Matemáticas. Proceedings de la XIV Simposio en Informática Educativa, 251-254.

Theodoridou, K. \& Yerasimou, T. (2008). Learning Spanish with Laura: the role of an Intelligent Agent in a Spanish Language Course, Proceedings of the ED-MEDIA conference, AACE.

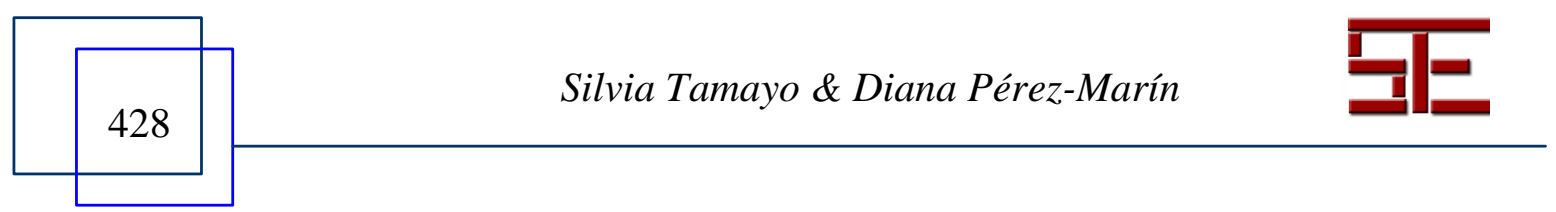




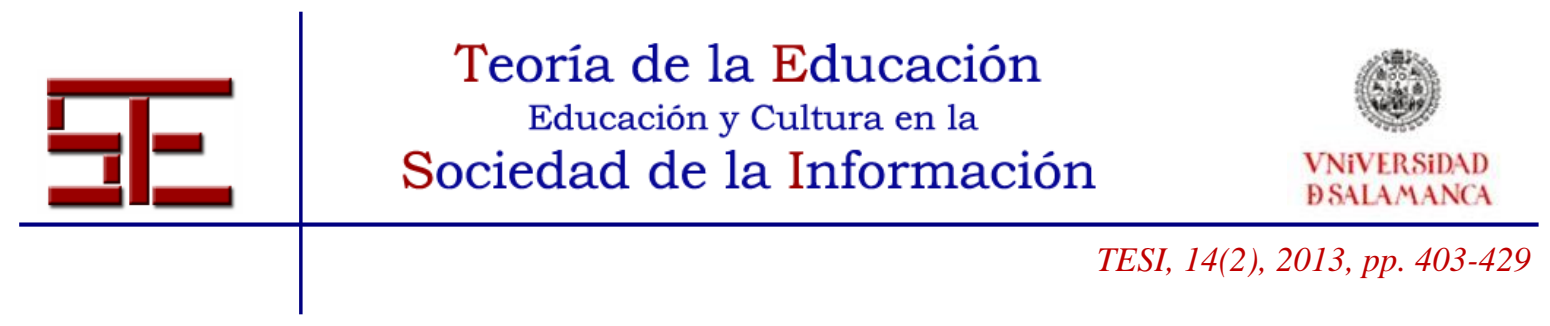

Wright, C. (1996). Learning disabilities in mathematics', National Center for Learning Disabilities Inc. http://www. idonline. org.

Yee, N., \& Bailenson, J. (2007). The Proteus effect: The effect of transformed selfrepresentation on behavior, Human Communication Research 33, 3.

Para citar el presente artículo puede utilizar la siguiente referencia:

Tamayo, S. y Pérez-Marín, D. 2013). Análisis de la experiencia de uso de un agente de comprensión lectora con niños en edad escolar. Revista Teoría de la Educación: Educación y Cultura en la Sociedad de la Información. 14(2), 403-429 [Fecha de consulta: dd/mm/aaaa].

http://campus.usal.es/ revistas_trabajo/index.php/revistatesi/article/view/10237/10670

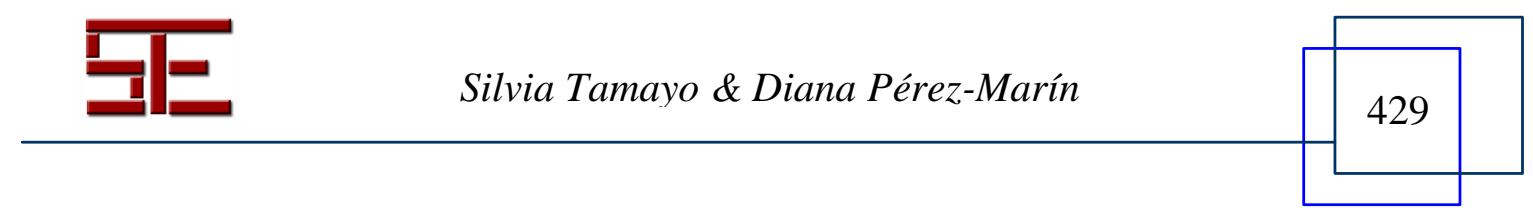

